TITLE:

\title{
Standing pulse-like solutions of a spatially aggregating population model(Dissertation_全文)
}

AUTHOR(S):

Ikeda, Tsutomu

CITATION:

Ikeda, Tsutomu. Standing pulse-like solutions of a spatially aggregating population model. 京都大学, 1985, 理学博士

\section{ISSUE DATE:}

1985-01-23

URL:

https://doi.org/10.14989/doctor.r5448

RIGHT: 


\begin{tabular}{|l|}
\hline 麻 制 \\
\hline 㼭 \\
\hline 482 \\
\hline \\
\hline 京大附図 \\
\hline
\end{tabular}

StANDING PULSE-LIKE SOLUTIONS

OF A SPATIALLY AGGREGATING POPULATION MODEL

\section{池田勉}




\section{学位蕃查報告}

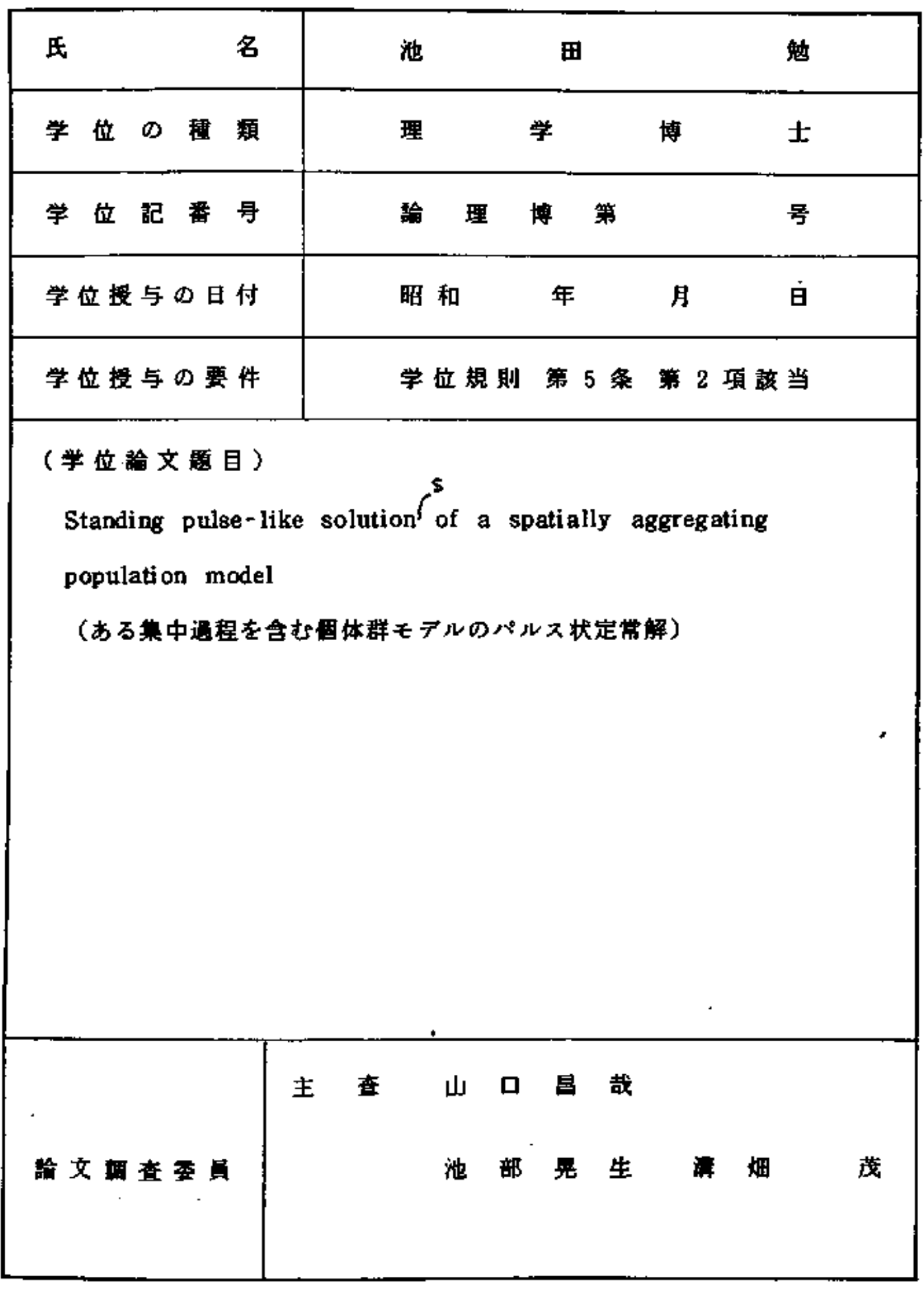

理学研究科 


\section{氏 名 池田勉}

\section{（链文内容の要旨）}

生物圈体群の数理モデルのーつに個体群のパターン形成を数学的に説明 しようとしてっくられた非線型微分程分方程式がある。この方程式は退化 型の拡散項上生物の图体の求心本能よりくる集中遇程をあらわす項と加ら なり，u時刻 $\mathrm{t}$ と位目 $\mathrm{x}$ の関数としての個体群密度しして，次の上うに 草ける。

$$
u_{i}(x, t)=\left(u^{m}\right)_{x x}(x, t)+\left[u(x, t)\left\{\int_{x-r}^{x} u(y, t) d y-\int_{x}^{x+r} u(y, t)\right\}\right]_{x}
$$

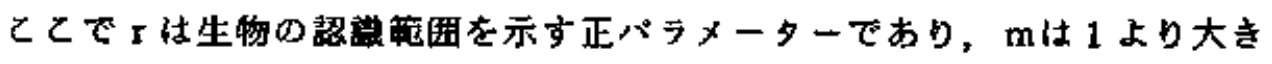
な数である。

申請者はこの方程式の定常解について次のような結果を得た。ここで \|\|$_{\infty}$ は $\mathrm{L}^{\infty}$ はノルム, \|\|$_{1}$ は $\mathbf{L}$ ノルムをらわすこととする。

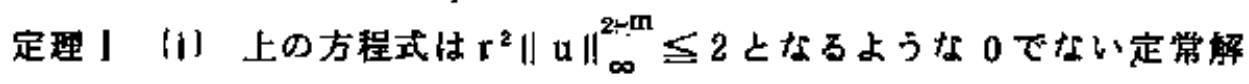

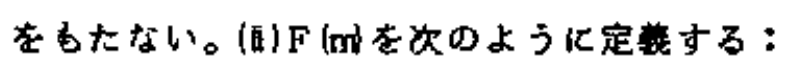

$\mathrm{F}(\mathrm{m})=2^{i-2 / \mathrm{m}} f^{\prime}\{\eta(1-\eta)\}^{-1 / m} \mathrm{~d} \eta \varepsilon$, 各正数 $\mathrm{q} \geq \mathrm{F}(m)^{2} k$ 対して, 上D 方程式は $\mathrm{r}^{2}\|\mathrm{u}\|_{\infty}^{2-\mathrm{m}_{\mathrm{I}} \mathrm{q}}$ であり，uの台の長さが $\mathrm{r}$ 以下の0でない定常解 を必らずるつ。この定常搨は台の内部で正镇であり台は連結である。この ような定常解を申請者は Standing Solitary Pulse とよんだ。(ili)爾数 $\mathrm{u}(\mathrm{x}) か ゙$ 上の方程式の0でない定常解ならは， u(x)は上述の Standing Solitary Pulse 隹よって展開できる。すなわち。

$$
\mathrm{u}(\mathrm{x})=\sum_{\mathrm{i} \in A} \mathrm{u}_{\mathrm{i}}(\mathrm{x})
$$

ここに $\left\{\mathbf{u}_{\mathrm{i}}\right\}_{\mathrm{i} \in A}$ は次の条件をみたす有限又は可算無限偲の Standing Solitory Pulse 列である:

$\left\{\begin{array}{l}\sup _{\substack{i \in A \\ i \neq j}}\left\{\left\|u_{i}\right\|_{\infty}\right\}<+\infty, \sum_{i \in A}\left\|u_{i}\right\|_{1}<+\infty \\ \left.i u_{i} \text { よ } u_{j} \text { の台の间の距炷 }\right) \geqq r\end{array}\right.$ 


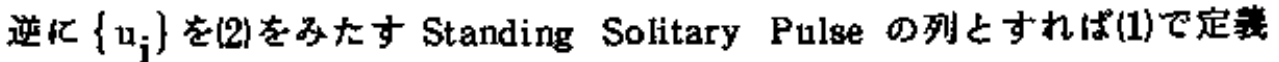
される $\mathrm{u}[\mathrm{x}]$ は上の方程式の0でない定常解である。

更に $\mathrm{m}=2$ の堨合火は更汇詳しい次の結果が得ら机ている。定理】は次 のように述べられる。

(i) $r^{2} \leqq 2$ ない゙0以外汇定常解をむたない。

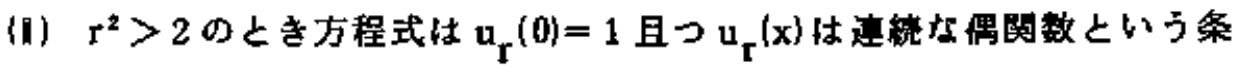
件をみたす唯一つの Standing Solitary Pulse 叫 $(x)$ を定常解としていつ。 この定常解 $u_{\mathbf{r}}$ の台はコンバクトであり，その長さを 方関数 $\mathrm{u}(\mathrm{x})$ が方程式の 0でない定常解となるための必罗十分条件は，上の Standing Solitary Pulse $u_{r}$ 有限因又は可算固の平行移较からなバル

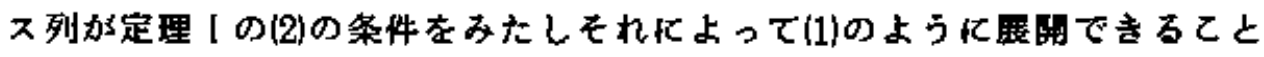

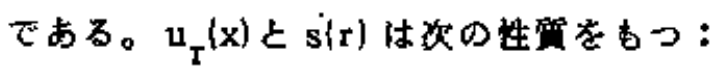

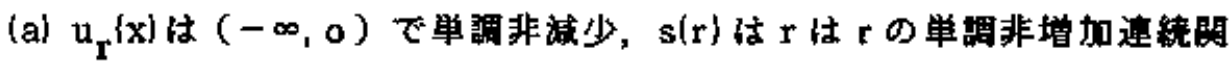

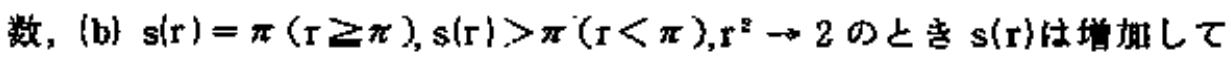

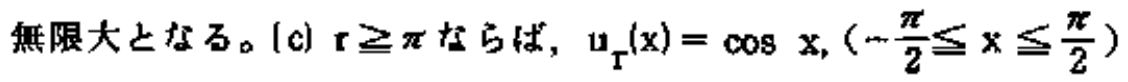

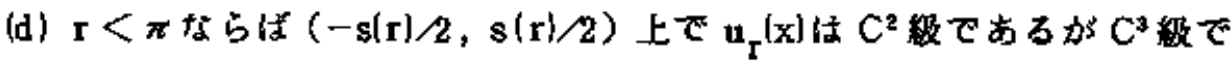
はない。

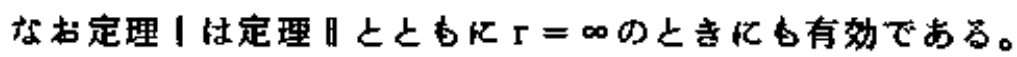




\section{怎名池田超}

\section{（路文番查の站果の要旨）}

申腤者の主部文は集中過程を含む，非線型搪散方程式で表現された生物 因体群のバターン形成についての数学的研究である。乙の看の方程式の研

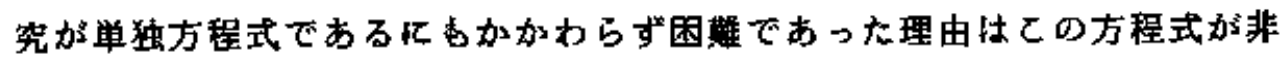

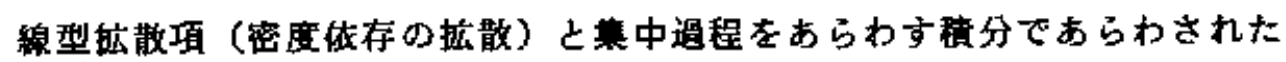

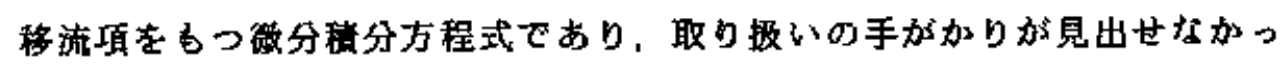
たことによっている。特别な場合 $\mathrm{r}=0$ の婸合杜は，方程式は多孔物䝷中 を摭数する流れの方程式こなり，䞍分項は消えるので Aronson の数篇の

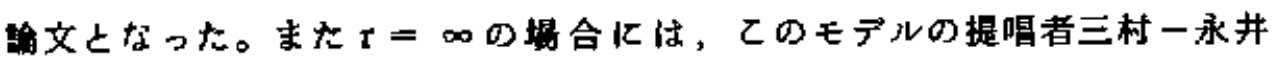

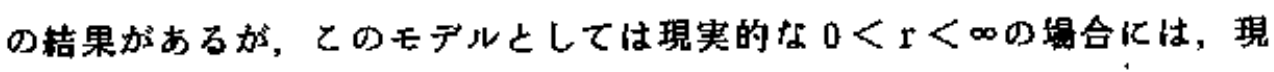

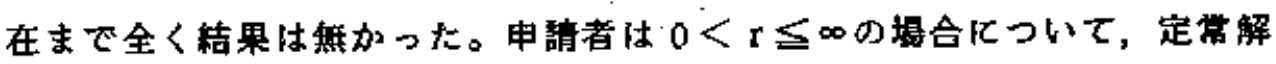

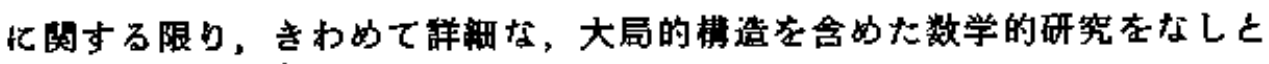

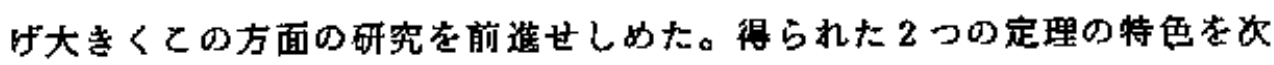
のようにまとめることができる。

1) 非自明な定常解の存在の条件を明示したてと。

2) 台肪连結で台の内では正值な定常解在 Standing Solitary Pulse となづけ，とれ定常解の最小単位としてとりあげ，その存在の条件 $\ell$, 任意の非自明定常解はてのような Standing Solitary Pulse $の$ 列机よって展開できるととを示したこと。又逆机そのような展開がて きる閔数住定常解であることを示した。

3） $m=2$ の婸合には更に詳細に上に述べた展開のための列は唯一つの 特别な形の Standing Solitary Pulseの有限又は可算调の平行移都に よって得られるととを示した。

4) $\mathrm{m}=2$ のときはバルスの間融と大きさ忆ついて, 正碎な定且的轺果 を得ていること。

5)証明の方法も新しく，たとえば 2 階の微分と、2 階の差分とのバラ 
ンスを研究するなど独剿的なとてろが多い。

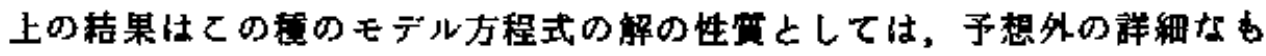

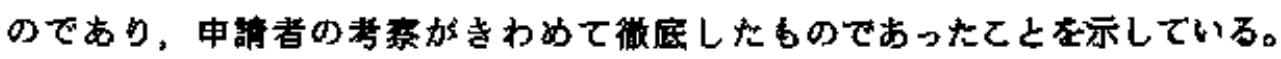
参考文 1，2.3 は非線型㣂微分方程式の差分化の研究であり，4.5.6 は申請者の得意とする移流項の加った迲散方程式の差分化の研究であり， 特に 6 は亚要なたノグラフである。7 は主給文の散散化の研究であり，い づれる申請者の学鿁の溧さを示するのである。

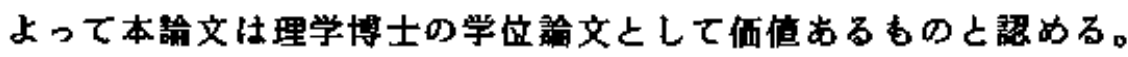

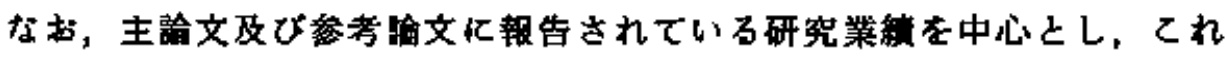
に関臬した研究分野について試問した結果，合格と熹めた。 
STANDING PULSE-LIKE SOLUTIONS

OF A SPATIALLY AGGREGATING POPULATION MODEL

Tsutomu Ikeda

Department of Mathematics

Faculty of Science

Kyoto University

Kyoto, Japan 
STANDING PULSE-LIKE SOLUTIONS

OF A SPATIALLY AGGREGATING POPULATION MODEL

Tsutomu Ikeda

Department of Mathematics

Faculty of Sclence

Kyoto University

Kyoto, Japan 
Abstract. The present paper is devoted to the study of stationary solutions of a nonlinear degenerate diffusion equation lnvolving a nonlocal convection term, which represents a mathematical model for spatially aggregating phenomena of populations. The equation has two ecological parameters: $m>1$ for the diffusion process and $0 \leq I$ $\leq \infty$ for the aggregating process expressed by the convection term. For the special case of $m=2$, this paper gives all stationary solutions of the one-parameter family $\{P(2, r)\}$ of the equations. The result asserts that $P(2, r)$ has no non-trivial stationary solution when $0 \leq r \leq \sqrt{2}$, while $P(2, r)$ has many pulse-11ke stationary solutions when $r>\sqrt{ } 2$. The paper also states a partial result for the general case of $\mathrm{m}$, and offers a view of the global structure of stationary solutions of $P(m, r)$.

Key words and phrases. Stationary solution, Nonlocal interaction, Density-dependent dispersal, Free boundary problem, Eigenvalue problem of differential-difference type.

Running head. A spatially aggregating population model. 


\section{Introduction}

In the present paper, we study the stationary solution of the following nonlinear degenerate diffusion equation involving a nonlocal convection term

$$
\begin{array}{r}
u_{t}(x, t)=\left(u^{m}\right) x_{x}(x, t)+\left(u(x, t)\left(\int_{x-r}^{x} u(y, t) d y-\int_{x}^{x+r} u(y, t) d y\right\}\right)_{x} \\
\text { in } R \times(0, \infty),
\end{array}
$$

where $m>1$ and $0 \leq r \leq \infty$ are parameters and $u(x, t) \geq 0$ denotes the population density at position $x \in R$ and time $t>0$. We denote the above equation by $P(m, r)$, by taking into account the parameters $m$ and $\mathbf{r}$.

Before the mathemattcal study of $P(m, r)$, we consider an ecologlcal meaning of the equation.

Ecology has become a mathematical subject in recent years. There have been proposed many spatially spreading population models for the study of the spatial distribution of organisms, dispersal of insects, migration of animals, plankton patchiness, coexistence of competing species, etc. (Okubo [36] among others). These spatially spreading population models, which are usually given by partial differential equations, include biological interactions and diffusion processes due to a spatial inhomogeneity in the density distribution; convection effects caused by a spatial inhomogeneity in environment also are included in several models.

We here note that some animals in näture congregate and form 
groups having clear interfaces (insect swarms and bird flocks for instance), and we proceed to the study of a mathematical model for spatially aggregating phenomena of organisms. Among others (for instance, a mathematical model for the aggregation of amoebae (Keller and Segel (24]) and the spatial pattern of amoeba aggregation (Sperb (421)), we are concerned with a class of spatially aggregating population models, proposed by Nagai and Mimura $\{32]$,

$$
\begin{array}{r}
u_{t}(x, t)=\left(u^{m}\right) x_{x x}(x, t)+\left(u(x, t) \int_{-\infty}^{\infty} k(x-y) u(y, t) d y\right)_{x} \\
\ln R \times(0, \infty),
\end{array}
$$

where each $K(x)$ is an odd function such that

$$
K(x) \geq 0 \quad \text { for } x>0
$$

The second term on the right-hand side of (1.1) ecologically shows an aggregating mechanism of individuals, which is motivated by the notion of "centripetal instincts" (Hamilton (19)). In fact, the term provides a mechanism that moves individuals at posttion $x$ to the right (resp. left) direction when

$$
\int_{0}^{\infty} x(y)(u(x+y, t)-u(x-y, t)) d y>0 \quad(\text { resp. }<0)
$$

since $\int_{0}^{\infty} K(y)\{u(x+y, t)-u(x-y, t)\} d y=-\int_{-\infty}^{\infty} K(x-y) u(y, t) d y$ represents the velocity of drift at position $x$. The first term on the righthand side of $(1.1)$ corresponds to the transport of population through a nonlinear diffusion process called density-dependent dispersal (Gurney and Nisbet [16] and Gurtin and MacCamy [17]). We note that the diffusion speed $m u^{m-1}$ decreases with the density $u$ and vanishes at position $x$ where $u(x)=0$. Consequently, the first term yields a homogenizing process while the second term yields a 
dehomogenizing process; a dellcate balance between these two processes may give rise to a spatial pattern that shows an aggregation of individuals.

The equation $P(m, r)$ is a special case of $(1,1)$, where the integral kernel $K(x)$ is given by

$$
K(x)=\left\{\begin{array}{cl}
1 & \text { for } 0<x<r, \\
-1 & \text { for }-r<x<0 \\
0 & \text { otherwise. }
\end{array}\right.
$$

Thus a non-trivial stationary solution of $P(m, r)$ ecologically exhibits a spatially aggregating pattern of individuals.

As stated in Nagai [31], mathematical models providing nonlocal interactions such as (1.1) are utilized to explain physical phenomena in other fields (Kuramoto [26] and Munakata (30)).

We now return to the mathematical discussion on the stationary solution of $P(m, r)$. (Later in this section, we will briefly review mathematical works related to the Cauchy problem (1.1) subject to a non-negative initial condition.)

The trivial function $u \equiv 0$ is always a stationary solution of $P(m, r)$, and we naturally are concerned with the non-trivial stationary solutions.

All stationary solutions of $P(m, 0)$ and $P(m, \infty)$ have already been obtained. The equation $P(m, 0)$ agrees to the porous medium equation (1.3)

$$
u_{t}=\left(u^{m}\right)_{x x} \quad \text { in } R x(0, \infty) \text {, }
$$

which appears in the theory of fluld flow through a porous medium 
(Bear [B] and Scheidegger (40]). Clearly, (1.3) has no non-trivial stationary solution. On the other hand, $P(m, \infty)$ has non-trivial standing solitary wave solutions with connected compact support (Mimura and Yamaguti [29]). These solutions are determined by the total population and the position of the center of support. In Section 8 , we shall discuss these stationary solutions in detail.

In the present paper, we shall study the stationary solution of $\mathrm{P}(\mathrm{m}, \mathrm{x})$ for $0<\mathrm{r}<\infty$.

First we shall deal with the special case of $m=2$. The result is summarized as

\section{Theorem 1.1 (Stationary solutions of $P(2, r)$ ).}

(I) Let $r \leq \sqrt{ }$. Then $P(2, r)$ has no non-trivial stationary solution.

(II) Let $r>\sqrt{ }$. Then $P(2, r)$ has a unique stationary solution $u_{r}$ such that

$$
\left\{\begin{array}{l}
u_{r} \text { is a continuous even function, } \\
u_{r}(0)=1, \\
u_{r} \text { has a connected compact support and is positive on the } \\
\text { interior of the support. }
\end{array}\right.
$$

Moreover, a function u becomes a non-trivial stationary solution of $P(2, r)$ if and only if $u$ is expressed in the form

$$
u(x)=\sum_{i \varepsilon \Lambda} a_{i} u_{x}\left(x-c_{i}\right) \text {, }
$$

where $\left\{a_{i}\right\}_{i \varepsilon \Lambda}$ and $\left\{c_{i}\right\}_{i \varepsilon \Lambda}(\Lambda:$ an index set) are finite or countable sequences of real numbers satisfying the conditions
$(1.6)$
$a_{1}>0$ for all i $\in \Lambda$.
$\sum_{i \in \Lambda} a_{i}<\infty$, 
$\left|c_{i}-c_{j}\right| \geq r+\left(\right.$ the length of the support of $u_{r}$ ) for all $i \in \Lambda, j \varepsilon \Lambda, i \neq j$.

(See Figure 1.1.) The length $s(r)$ of the support of $u_{r}$ has the properties

(2) $s(r)$ increases with decreasing $r$,

(3) $s(r)$ is continuous in $(\sqrt{2}, \infty)$,

(4) $s(r)$ tends to infinity as $r$ tends to $\sqrt{ } 2$.

The stationary solution $u_{r}$ has the properties

(i) $u_{r}$ is non-decreasing on $(-\infty, 0)$,

(ii) for $r \geq \pi, u_{r}(x)=\cos x$ on $\left(\frac{\pi}{2}, \frac{\pi}{2}\right) \equiv\left(-\frac{1}{2} s(r), \frac{1}{2} s(r)\right)$,

(iii) for $\sqrt{ } 2<r<\pi, u_{r}$ is twice continuously differentiable on $\left(-\frac{1}{2} s(x), \frac{1}{2} s(r)\right)$ but $u_{r}$ is not thrice continuously differentiable on $\left(-\frac{1}{2} s(r), \frac{1}{2} s(r)\right)$. I

We need the condition $(1.6)$ since the stationary solution of $P(m, I)$ is defined as a non-negative valued function belonging to $L^{\infty}(R)$ and $L^{1}(R)$ (Section 2). The condition (1.7) asserts that the distance between two arbitrary connected components of the support of a stationary solution is greater than or equal to $r$. For instance, $d_{1} \geq r$ and $d_{2} \geq r$ in Figure 1.1 . We note that Theorem 1.1 is valid for all $0 \leq r \leq \infty$; when $r=\infty$, the support of a stationary solution should be connected by (1.7) and its length equals $\pi$ by the property (1) of $s(r)$.

We shall show, in Section 3 , that all stationary solutions of $P(m, r)$ are decomposed into simple ones, each of which has a connected support and 1 s positive on the interior of its support. In accordance with Nagai and Mimura [33], we refer to these simple 
stationary solutions as standing solitary pulses. The stationary solution $u_{r}$ is a standing solttary pulse. A standing solitary pulse becomes a solution of a free boundary problem of differentialdifference type (Section 4). This free boundary problem is reduced to a linear one when $m=2$, and we can characterize the stationary solution $u_{r}$ by using a principal eigenfunction of an eigenvalue problem derived from the free boundary problem (Sections 5 to 7 ).

Furthermore, we shall deal with the general case of $m \neq 2$ (Section 8). The above method of proof cannot apply to the general case since the free boundary problem becomes a nonlinear one when m ₹2. We have not yet obtained a satisfactory result. However, we can extend, to the general case, the first assertion in Theorem 1.1 and the fact that $P(2, r)$ has a stationary solution such that the length of its support is less than or equal to $r$ when $r \geq \pi$. (Observe the property (1) of $s(x)$.

Theorem 1.2 (Stationary solutions of $P(m, r)$ for $m \neq 2$ ). (I) $P(m, r)$ has no non-trivial stationary solution $u$ such that

$$
r^{2}\|u\|_{\infty}^{2-m} \leq 2 \text {, }
$$

where $\|\cdot\|_{\infty}$ denotes the supremum norm.

(II) Let $F(m)=2^{1-2 / m} \int_{0}^{1}(\eta(1-\eta))^{-1 / m} d \eta$ for $m>1$. For each positive number $q$ such that

$$
\text { (1.9) } \quad q \geq F(m)^{2},
$$

$P(m, r)$ has a stationary solution u such that

$$
r^{2} \mid u \|_{\infty}^{2-m}=q
$$


and the length of the support of $u$ is less than or equal to $r$. (See Figure 8.1.) I

The conditions $(1.8)$ and $(1.9)$ with $(1.10)$ are reduced to

$$
r \leq \sqrt{2} \text { and } \quad I \geq \pi \text {, }
$$

respectively, when $m=2$. Hence Theorem 1.2 is a partial extension of Theorem 1.1. We shall also give a conjecture for the global structure of stationary solutions of $P(m, r)$ in section 8 .

We here review mathematical works related to the Cauchy problem (1.1) subject to a non-negative initial condition.

When the integral kernel $\mathrm{K}=0,(1.1)$ is reduced to the porous medium equation (1.3). The distinctive feature of (1.3), which is caused by the degeneracy of diffusion at $u=0$, is that an initial distribution with compact support spreads out at a finite speed (Oleinik et al. [37]) and loges its initial smoothness (Aronson [4] and Kalashnikov [22]). We can observe this feature in an exact solution obtalned by Barenblatt [7] and Pattle [38]. A similar exact solution of $u_{t}=\left(u^{2}\right)_{x x}+u(1-u)$ was found by Newman [35]. A solution of the Cauchy problem (1.3) is therefore defined in a generalized sense, and the regularity of the generalized solution is discussed in Aronson [3], Caffarelli and Friedman [9], Gilding and Peletier [14], etc. Because of the finite propagation property, there appear interface curves that divide the half plane $B \times(0, \infty)$ into two regions $\{(x, t) ; u(x, t)>0\}$ and $\{(x, t) ; u(x, t)=0\}$ (for instance, Aronson [5], Caffarelli and Friedman [10] and Knerr [25]). Graveleau and Jamet [15] and Tomoeda and Mimura [43] have proposed 
finite difference schemes where the degeneracy of diffusion is taken Into account. Schemes proposed by DiBenedetto and Hoff [12], Mimura et al. [28] and Hoff [20] approximate the interface curves as well as the value of the unknown function.

The Cauchy problem (1.1) has the same distinctive property as that of $(1.3)$. For a class of integral kernels $\mathrm{K}$ including the simple one given by $(1.2)$, Nagal $\{31]$ has shown the unique existence and the regularity of the generalized solution of $(1.1)$, and he has proved the finite propagation property. Nagai and Mimura [33] have obtained the asymptotic behavior of the solution of $P(\mathrm{~m}, \infty)$ : as time $t$ tends to infinity, a solution of $\mathrm{P}(\boldsymbol{m}, \infty)$ tends to a stationary solution uniquely determined by the initial condition. Recently, Nagai and Mimura [34] have derived an equation that describes the motion of the interfaces to $P(m, \infty)$, and have obtained the asymptotic behavior of the interfaces. Ikeda [21] has devised a finite difference scheme for $P(2, \infty)$ and has obtained the asymptotic behavior of the discrete solution. (Figure 1.2 displays a solution of the Cauchy problem $P(2, \infty)$ obtained by this scheme.) A modification of this scheme has been useful in the study of the stationary solution of $P(2, r)$.

Alt ([1] and [2]) has studied stable patterns reproduced by another spatlally aggregating population model providing both a degenerate diffusion process and an aggregating process expressed by a nonlocal interaction. 


\section{Regularity of a stationary solution}

In this section, we first define a stationary solution of $P(m, r)$ and then consider its regularity.

A stationary solution $u$ of $P(m, r)$ is defined to be a non-negative valued function belonging to $L^{1}(R)$ and $L^{\infty}(R)$ that satisfies

$$
\text { (2.1) } \quad\left\{\left(u^{m}\right)_{x}+u\left\{\int_{x-r}^{x} u d y-\int_{x}^{x+r} u d y\right\}\right\}_{x}=0
$$

In the distribution sense. (We denote by $C_{0}^{\infty}(\Omega)$ the space of infinitely differentiable functions defined on an open subset $\Omega$ of $R$ having compact support in $\Omega .1$

Let $u$ be a stationary solution of $P(m, r)$. An application of the distribution theory yields that the distribution

$$
F[u] \equiv\left(u^{m}\right) x+u\left(\int_{x-r}^{x} u d y-\int_{x}^{x+r} u d y\right\}
$$

is a constant $C$, and that this constant $C$ is represented by

$$
C=F[u](\phi) \equiv\langle F[u], \phi\rangle
$$

with $\phi \varepsilon C_{0}^{\infty}(R)$ such that $\int_{-\infty}^{\infty} \phi(x) d x=1$ (Schwartz $\left.(41, p p .51-53]\right)$. The constant $C$ equals zero by the following proposition.

Proposition 2.1 (Regularity of a stationary solution). If u is a stationary solution of $P(m, r)$, then

$$
u \varepsilon C(\mathbf{R}) \text { and } u^{m} \varepsilon C^{1}(R) \text {, }
$$

and $\mathrm{u}$ satisfies

$$
\text { (2.2) } \quad F[u] \equiv\left(u^{m}\right)_{x}+u\left\{\int_{x-r}^{x} u d y-\int_{x}^{x+r} u d y\right\}=0 \text { in } R
$$


in the classical sense. Moreover, $u$ is twice continuously differentiable on the region $D_{u}=\{x \varepsilon R ; u(x)>0\}$.

Proof. We fix a function $\phi \varepsilon C_{0}^{\infty}(R)$ satisfying $\int_{-\infty}^{\infty} \phi(x) d x=1$, and define by $\Phi_{n}(x)=\frac{1}{n} \phi\left(\frac{x}{n}\right)$ a sequence $\left\{\phi_{n}\right\}_{n=1}, 2, \ldots, \Phi_{n} \varepsilon C_{0}^{\infty}(R)$ and $\int_{-\infty}^{\infty} \Phi_{n}(x) d x=1$. Since $u$ belongs to $L^{1}(R)$ and $L^{\infty}(R)$, the constant $C=\langle F[u], \phi\rangle=\left\langle F[u], \phi_{n}\right\rangle$ is estimated by

$$
\begin{aligned}
|c| & \leq\left|\left\langle u^{m},\left(\Phi_{n}\right)_{x}\right\rangle\right|+\mid\left\langle u\left\{\int_{x-r}^{x} u d y-\int_{x}^{x+r} u d y\right\}, \Phi_{n}>\right| \\
& \leq\left\|u^{m}\right\|_{\infty}\left\|\left(\Phi_{n}\right)_{x}\right\|_{1}+\|u\|_{1}^{2}\left\|\Phi_{n}\right\|_{\infty} \\
& =\frac{1}{n}\left\{\left\|u^{m}\right\|_{\infty}\left\|\Phi_{x}\right\|_{1}+\|u\|_{1}^{2} \| \phi_{\infty}\right\} \downarrow 0 \text { as } n \uparrow \infty,
\end{aligned}
$$

which implies that $C$ equals zero. (The symbol $\|\cdot\|_{1}$ denotes the usual norm of $\left.L^{1}(R).\right) \quad$ since $F[u] \equiv 0,\left(u^{m}\right)_{x}$ is represented by

$$
\left(u^{m}\right)_{x}=-u\left(\int_{x-r}^{x} u d y-\int_{x}^{x+r} u d y\right\}
$$

The right-hand side of $(2.4)$ belongs to $L^{1}(R)$ and $L^{\infty}(R)$, hence $u^{m} \varepsilon$ $C(R)$, and $u \in C(R)$. We know that $u^{m} \in C^{1}(R)$ by using (2.4) once again. Hence u satisfles (2.2) in the classical sense.

\section{In general,}

(2.5) $\left\{\begin{array}{l}\text { if } f \in C^{1}(G) \text { and } f>0 \text { on } G \text { (G: an open set), } \\ \text { then } f^{\alpha} \varepsilon C^{1}(G) \text { for all } \alpha \varepsilon R \text {. }\end{array}\right.$

Applying (2.5) to the case where $G=D_{u}, f=u^{m}$ and $\alpha=1 / m$, we know that $u=\left(u^{m}\right)^{1 / m}$ is continuously differentiable on $D_{u}$. Now (2.4) yields that $\mathrm{u}^{\mathrm{m}}$ is twice continuously differentiable on $\mathrm{D}_{\mathrm{u}}$ since $\int_{x-r}^{x} u d y-\int_{x}^{x+r} u d y E C^{1}(R)$. Hence $u$ is twice continuously differentiable on $D_{u}$ since $u_{x}=\frac{1}{m^{1-m}}\left(u^{m}\right)$ on $D_{u}$ and $u^{1-m}$ is continuously 
differentiable on $D_{\mathrm{u}}$ by $(2.5)$. I

A stationary solution $u$ of $P(m, \infty)$ is infinitely differentiable on $D_{u}=\{x \in R ; u(x)>0\}$ (Mimura and Yamaguti $\left.\{29\}\right)$. When $0<r<$

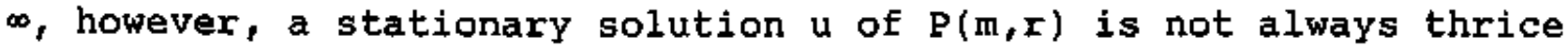
continuously differentiable on $\mathrm{D}_{\mathrm{u}}$. In fact, a non-trivial stationary solution $u$ of $P(2, r)$ is never thrice continuously differentiable on $D_{u}$ when $r<\pi$, while $u$ is infinitely differentiable on $D_{u}$ when $r$ $\geq \pi$ (Theorems 1.1 and 5.9 ).

As stated in section 1 , a stationary solution $u$ of $P(m, r)$ is called "a standing solitary pulse" if $u$ has a connected support and 1s positive on the interior of its support. 


\section{Decomposition of a stationary solution}

In this section, we discuss the support of a stationary solution of $\mathrm{P}(\mathrm{m}, \mathrm{r})$ and decompose a stationary solution into the standing solitary pulses. The support of a continuous function $\mathrm{v}$ is denoted by supp[v]. A standing solitary pulse is abbreviated to an "sspulse".

Even if both $u$ and $v$ are ss-pulses of $P(m, r), u+v$ is not always a stationary solution of $\mathrm{P}(\mathrm{m}, \mathrm{r})$, of course. However, if the distance between supp $[u]$ and supp $[v]$ is greater than or equal to $r$, then $F[u+v]=F[u]+F[v]((2.2)$ in Proposition 2.1) and $u+v$ becomes a stationary solution of $P(m, r)$.

The following theorem asserts that all non-trivial stationary solutions of $P(m, r)$ are obtained by adding ss-pulses in the above way:

Theorem 3.1 (Decomposition of a stationary solution). All non-trivial stationary solutions $u$ of $P(m, r), r>0$, are expressed in the form

$$
u=\sum_{i \varepsilon \Lambda} u_{i}
$$

where $\left\{u_{i}\right\}_{i \varepsilon \Lambda}(\Lambda:$ an index set) is a finite or countable sequence of ss-pulses such that

$$
\operatorname{dis}\left(\operatorname{supp}\left[u_{i}\right], \operatorname{supp}\left[u_{j}\right]\right) \geq r \text { for all } i \varepsilon \Lambda, J \varepsilon \Lambda, 1 \neq j \text {, }
$$

where $\operatorname{dis}(A, B)$ denotes the distance between subsets $A$ and $B$ of $R$.

Proof. The proof 1 s given later in this section. 1 
By virtue of Theorem 3.1, we have only to study the ss-pulse in the forthcoming sections.

We begin with a change of the unknown function. With a stationary solution $u$ of $P(m, r)$, we associate a function $U$ defined by

$$
U(x)=\int_{-\infty}^{x} u(y) d y \quad \text { for } x \in R \text {. }
$$

This function $\mathrm{U}$ has the following properties:

(3.2) $\left\{\begin{array}{l}U \varepsilon C^{1}(R), U \text { is non-decreasing on } R, \\ U(x) \downarrow 0 \text { as } x+-\infty \text { and } U(x)+\|u\|_{1} \text { as } x+\infty, \\ U \text { ts thrice continuously differentiable on } D_{u},\end{array}\right.$ where $D_{u}=\left\{x \in R_{;} u(x)>0\right\}$. By the function $U,(2.2)$ is rewritten as

(3.3) $\left\{U_{x}(x)^{m}\right\}_{x}-U_{x}(x)\{U(x+r)+U(x-r)-2 U(x)\}=0 \quad$ in $R$.

A primitive function $J[U]$ of the function on the left-hand side of (3.3) is given by

$$
J[U](x)=U_{x}(x)^{m}+\int_{x}^{x+r_{U}}(y)\{U(y-r)-U(x)\} d y
$$

$$
=u(x)^{m}+\int_{x}^{x+r} u(y)\{U(y-r)-U(x)\} d y \quad \text { for } x \in R \text {. }
$$

By (3.3), J[U] equals a constant $C$. Moreover, since $u \in L^{\infty}(R)$ by the definition of a stationary solution,

$$
\int_{x}^{x+I} u(y)(U(y-r)-U(x)\} d y \rightarrow 0 \text { as } x+\infty
$$

by $(3,2)$. Hence $\lim _{x+\infty} u(x)^{m}$ exists and equals $c$. Since $u^{m} \varepsilon L^{1}(R)$ by the definition of a stationary solution, $c=\lim _{x \rightarrow \infty} u(x)^{m}=0$, and

$$
J[U](x)=0 \quad \text { for } x \varepsilon R
$$


By using the function $J[U]$, we obtain

Lemma 3.2. Let $u$ be a stationary solution of $P(m, r)$. If $u(y)=0$ at a point $y \varepsilon R$, then there exists a closed interval I such that $y \in I$, (the Iength of $I$ ) $\geq r$ and $u(x)=0$ for $x \varepsilon I$.

Proof. Let $a=\inf \{x \in\{y-r, y] ; u=0$ on $\{x, y]\}$ and $b=$ $\sup \{x \in\{y, y+r] ; u=0$ on $[y, x]\}$. It suffices for the proof to show that $r \leq b-a$. suppose that $r>b-a$. Let $U$ be a nondecreasing function defined by (3.1). By the assumption,

$$
U(x)<v(a) \text { for } x<a
$$

and there exists a real number $c$ such that

$$
b<c<a+r \text { and } U_{x}(x)=u(x)>0 \text { for } b<x<c \text {. }
$$

These together with (3.2) lead to the estimate

$$
\begin{aligned}
J[U](a) & =u(a)^{m}+\int_{a}^{a+r} u(x)\{U(x-r)-U(a)\} d x \\
& =\int_{b}^{a+r} u(x)\{U(x-r)-U(a)\} d x \leq \int_{b}^{c} u(x)\{U(x-r)-U(a)\} d x<0,
\end{aligned}
$$

which contradicts (3.5). Thus the proof is completed. I

Lemma 3.2 enables us to prove Theorem 3.1 .

Proof of Theorem 3.1. Let $u$ be a non-trivial stationary solution of $P(m, I), r>0$. Lemma 3.2 shows that the distance between two arbitrary connected components of supp[u] is greater than or equal to $r$. This implies that the number of connected components of supp [u] is finite or countable. Let $\left\{S_{i}\right\}_{i \varepsilon \Lambda}$ be the sequence of connected components of supp[u] $\left(\Lambda:\right.$ an index set). With each $s_{i}(i$ E 1 ), we associate a function $u_{1}$ given by

$$
u_{i}(x)=u(x) \text { for } x \in s_{1} \text { and } u_{i}(x)=0 \text { for } x \neq s_{i} \text {. }
$$


Then, $u(x)=\sum_{i \varepsilon \Lambda} u_{i}(x)$ for $x \in R$, and each $u_{i}$ is positive on the interior of $s_{i}$ by Lemma 3.2. Now it suffices to show that each $u_{i}$ becomes a stationary solution of $P(m, r): F\left[u_{1}\right]=0$ on $R((2.2)$ in Proposition 2.1). Since $u_{i}=0$ on $R \backslash S_{i}, F\left[u_{i}\right](x)=0$ for $x i S_{i}$. Lema 3.2 implies

$$
u(y)=u_{i}(y) \text { for } y \in R \text { such that } \operatorname{dis}\left(\{y\}, s_{i}\right) \leq r ;
$$

hence $F\left[u_{i}\right](x)=F[u](x)=0$ for $x \& s_{i}$. We thus complete the proof of Theorem 3.1. I

Remark 3.3. Theorem 3.1 and Lemma 3.2 are valid for all $0 \leq r$ $\leqslant \infty$; hence a stationary solution of $\mathrm{P}(\mathrm{m}, \infty)$ has a connected support (M1mura and Yamaguti [29]). I

Remark 3.4. Let $u(x, t)$ be a solution of the cauchy problem $P(m, r)$ subject to a non-negative initial condition. Then the total population $\int_{-\infty}^{\infty} \mathrm{u}(\mathrm{y}, \mathrm{t}) \mathrm{dy}=\mid \mathrm{u}(\cdot, \mathrm{t}) \|_{1}$ is kept constant (Gilding [13]). Hence $P(m, r)$ is transformed into

$$
U_{t}(x, t)=\left\{U_{x}(x, t)^{m^{m}}\right\}_{x}-U_{x}(x, t)\{U(x+r, t)+U(x-r, t)-2 U(x, t)\}
$$

through the same change of the unknown function as (3.1). By J[U], this equation is expressed in the divergence form

$$
U_{t}(x, t)=J[U]_{x}(x, t) \text {. } \quad 1
$$

Remark 3.5. Let $u$ be a stationary solution of $P(m, r)$ and define a non-decreasing function $U$ by (3.1). The function $J[U]$ is rewritten as

$$
J[U](x)=U_{x}(x)^{m}-U(x)(U(x+r)-U(x))+\int_{x}^{x+r} U_{y}(y) U(y-r) d y .
$$

since $u=U_{x} \geq 0$ on $R, J[U]=0$ on $\mathbf{R}$ and the third term on the 
right-hand side of the above equality is non-negative by $(3.2)$,

$$
\|u\|_{\infty}^{m}=\sup _{x \in R}\left\{u(x)^{m}\right\}=\sup _{x E R}\left\{U_{x}(x)^{m}\right\}
$$

$$
\begin{aligned}
& \leq \sup _{x \varepsilon R}\{U(x)(U(x+r)-U(x))\} \\
& \leq \sup _{x \varepsilon R}\left\{U(x)\left(\|u\|_{1}-U(x)\right)\right\}=\left(\|u\|_{1} / 2\right)^{2} .
\end{aligned}
$$

When $r=\infty, U[U](x)$ is reduced to

$$
J[U](x)=U_{x}(x)^{m}-U(x)\left(\|u\|_{1}-U(x)\right),
$$

and $(3.6)$ is replaced by the equality

(3.7)

$$
\|u\|_{\infty}^{m}=\sup _{x \varepsilon \in R}\left\{u(x)^{m}\right\}=\sup _{x \varepsilon R}\left\{U_{x}(x)^{m}\right\}
$$

$$
=\sup _{x \varepsilon R}\left\{U(x)\left(\|u\|_{1}-U(x)\right)\right\}=\left(\|u\|_{1} / 2\right)^{2} . \quad \text { I }
$$




\section{Standing solitary pulses}

In Sections 4 to 6 , we study the ss-pulse of $P(2, r)$.

The present section gives a linear differential-difference equation that the ss-pulse satisfies in the interior of its support. By using this linear equation, we show that $P(2, r)$ has no nontrivial stationary solution when $r \leq \sqrt{2}$.

Let us be an ss-pulse of $\mathrm{P}(\mathrm{m}, \mathrm{r})$. Since $\left(\mathrm{u}^{\mathrm{m}}\right)_{\mathrm{x}}=\frac{\mathrm{m}}{\mathrm{m}-1} \mathrm{u}\left(\mathrm{u}^{\mathrm{m}-1}\right)_{\mathrm{x}}$ on the interior int (supp[u]) of $\operatorname{supp}[u]$, dividing $(2,2)$ by $u>0$, we obtain

$$
\text { (4.1) } \left.\quad \frac{m}{m-1}\left(u^{m-1}\right)_{x}+t \int_{x-r}^{x} u d y-\int_{x}^{x+r} u d y\right\}=0 \text { in } \operatorname{int}(\operatorname{supp}[u]) \text {. }
$$

Hence an ss-pulse $u$ of $P(m, r)$ satisfies the differentlal-difference equation (Hale [18] and Lange and Miura [27])

$$
\frac{m}{m-1}\left(u^{m-1}\right) x x-\{u(x+r)+u(x-r)-2 u(x)\}=0 \text { in } \operatorname{int}(\operatorname{supp}[u]) \text {, }
$$

which is obtained by differentiating (4.1). We note that

(1) both $(4.1)$ and $(4.2)$ are valid for all $m>1$,

(2) $u^{\mathbb{m}-1} \in \mathrm{H}^{1}(\mathrm{R})$ for all m $>1$,

(3) (4.2) is reduced to the linear equation when $m=2$.

We denote by $\Delta$ and $\nabla$, respectively, the Laplacian operator and the gradient operator:

$$
\Delta v \equiv v_{x x} \equiv(d / d x)^{2} v \text { and } \quad \nabla v \equiv v_{x} \equiv \frac{d v}{d x}
$$

We introduce a discrete Laplacian operator $\Delta_{b}$ and $a$ discrete gradient operator $\nabla_{b}$ given by the central finite difference approximation for $\Delta$ and $\nabla$, respectively: 


$$
\left\{\begin{array}{l}
\left(\Delta_{b} v\right)(x) \equiv\left(\frac{1}{b}\right)^{2}\{v(x+b)+v(x-b)-2 v(x)\}, \\
\left(\nabla_{b} v\right)(x) \equiv \frac{1}{b}\left\{v\left(x+\frac{b}{2}\right)-v\left(x-\frac{b}{2}\right)\right\} \quad \text { for } b>0 .
\end{array}\right.
$$

For $m=2$, the equation $(4.2)$ is now rewritten as

$$
\text { (4.3) } \quad \Delta u-\frac{1}{2} r^{2} \Delta_{r} u=0 \quad \text { in int (supp [u]). }
$$

The first and second terms on the left-hand side of (4.3) correspond to the nonlinear diffusion process $\left(\mathrm{u}^{2}\right)_{\mathrm{xx}}$ and the aggregating process $\left(u\left(\int_{x-r}^{x} u d y-\int_{x}^{x+r} u d y\right)\right)_{x}$, respectively. We note that the problen of the balance between the diffusion process and the aggregating process is reduced to that of the balance between the Laplacian operator and the discrete Laplacian operator when $m=2$. We also note that if $u(x)$ is an ss-pulse of $P(2, r)$, then so is au(x) (a: an arbitrary positive number) as well as any translation of $u(x)$.

By (4.3), an ss-pulse $u$ of $P(2, r)$ satisfies

$$
\left\{\Delta u-\frac{1}{2} x^{2} \Delta u\right\} v=0 \text { in } R
$$

for all $v \varepsilon H^{1}(R)$ such that $v=0$ on $R \backslash i n t(\operatorname{supp}[u])$. Integrating this equation on $R$, we obtain

$$
\text { (4.4) } \quad(\nabla u, \nabla v)-\frac{1}{2} r^{2}\left(\nabla_{r} u, \nabla_{r} v\right)=0
$$

for all $v \varepsilon H^{9}(R)$ such that $v=0$ on $R \backslash \operatorname{int}(\operatorname{supp}[u])$, where $(\cdot, \cdot)$ denotes the inner product of $L^{2}(R)$. (For brevity the norm of $L^{2}(R)$ is denoted by $1 \cdot$ in the present paper.)

Lemma 4.1. The Laplaclan operator is stronger than the discrete Laplacian operator in the sense that

$$
(\nabla v, \nabla v)>\left(\nabla_{b} v, \nabla_{b} v\right) \quad \text { for } a l l v \varepsilon H^{1}(R) \backslash(0) \text { and } b>0
$$

Proof. Hölder's inequality yields 


$$
|v(x+b)-v(x)|^{2}=\left|\int_{x}^{x+b} y_{y}(y) d y\right|^{2} \leq b \int_{x}^{x+b}\left|v_{y}(y)\right|^{2} d y
$$

In the above, the equality sign holds only when $v$ is constant on the interval $(x, x+b)$. Since $v \in H^{1}(R) \backslash\{0\}, V$ is not constant on $R$ and

$$
\begin{aligned}
\left(\nabla_{b} v, \nabla_{b} v\right) & =\left(\frac{1}{b}\right)^{2} \int_{-\infty}^{\infty}\left|v\left(x+\frac{b}{2}\right)-v\left(x-\frac{b}{2}\right)\right|^{2} d x=\left(\frac{1}{b}\right)^{2} \int_{-\infty}^{\infty}|v(x+b)-v(x)|^{2} d x \\
& <\frac{1}{b} \int_{-\infty}^{\infty} d x \int_{x}^{x+b}\left|v_{y}(y)\right|^{2} d y=\frac{1}{b} \int_{-\infty}^{\infty} d y \int_{y-b}^{y}\left|v_{y}(y)\right|^{2} d x \\
& =\int_{-\infty}^{\infty}\left|v_{y}(y)\right|^{2} d y=(\nabla v, \nabla v),
\end{aligned}
$$

which completes the proof. I

An ss-pulse $u$ of $P(2, r)$ must satisfy $(4.4)$. Hence Lemma 4.1 implies that $P(2, r)$ has no non-trivial ss-pulse when $I \leq \sqrt{ } 2$, Combining this fact and Theorem 3.1, we obtain

Theorem 4.2 (Non-existence of a stationary solution for $r \leq \sqrt{2}$ ). Let $r \leq \sqrt{ } 2$. Then the equation $P(2, r)$ has no non-trivial stationary solution. $\theta$

Lemma 4.3. Let $b$ and $c$ be positive numbers $(b \leq c)$. Then,

$$
\left\|\nabla_{b} v-\nabla_{c} v\right\| \leq \frac{2}{c}|c-b|\|\nabla v\| \quad \text { for all } v \in H^{1}(R)
$$

Proof. We put $B=b / 2$ and $Y=c / 2$. By Cauchy's inequality,

$$
\begin{aligned}
& \left(b c\left|\nabla_{b} v-\nabla_{c} v\right|\right)^{2}=\left|c \int_{x-\beta}^{x+\beta} v_{y}(y) d y-b \int_{x-\gamma}^{x+\gamma} \gamma_{y}(y) d y\right|^{2} \\
& =\left|(c-b) \int_{x-\beta}^{x+\beta} v_{y}(y) d y-b\left(\int_{x-\gamma}^{x-\beta} v_{y}(y) d y+\int_{x+\beta}^{x+\gamma} v_{y}(y) d y\right\}\right|^{2} \\
& \leq 2(c-b)^{2}\left|\int_{x-\beta}^{x+\beta} v y d y\right|^{2}+2 b^{2}\left|\int_{x-\gamma}^{x-\beta} v y d y+\int_{x+\beta}^{x+\gamma} v y d y\right|^{2} \\
& \leq 2(c-b)^{2}\left|\int_{x-\beta}^{x+\beta}{ }^{v} y d y\right|^{2}+4 b^{2}\left|\int_{x-\gamma}^{x-\beta} v_{y} d y\right|^{2}+4 b^{2}\left|\int_{x+\beta}^{x+\gamma} v d y\right|^{2} .
\end{aligned}
$$


Then Hölder's inequality yields

$$
\begin{aligned}
& \left\{b c\left|\nabla_{b} v-\nabla_{c} v\right|\right\}^{2} \leq 2 b|c-b|^{2} \int_{x-\beta}^{x+\beta}\left|v_{y}\right|^{2} a y \\
& +2 b^{2}|c-b| \int_{x-\gamma}^{x-\beta}\left|v_{y}\right|^{2} d y+2 b^{2}|c-b| \int_{x+\beta}^{x+\gamma}\left|v_{y}\right|^{2} d y \text {. }
\end{aligned}
$$

Now, by the same method as in the proof of Lemma $4.1,\left(b c\left\|\nabla_{b} v-\nabla_{c} v\right\|\right)^{2}$ is estimated by

$$
\begin{aligned}
\left(b c\left\|\nabla_{b} v-\nabla_{c} v\right\|\right\}^{2} & =\int_{-\infty}^{\infty}\left(b c\left|\nabla_{b} v-\nabla c v\right|\right\}^{2} d x \\
& \leq 2 b^{2}|c-b|^{2}\|\nabla v\|^{2}+b^{2}|c-b|^{2}\|\nabla v\|^{2}+b^{2}|c-b|^{2}\|\nabla v\|^{2} \\
& =\{2 b|c-b|\|\nabla v\|\}^{2} .
\end{aligned}
$$

This completes the proof. I

Lemma 4.4. Let $\{v, v\}$ be a pair of functions on $\mathbf{R}$ such that $v$ $\varepsilon C(R), V \varepsilon C^{1}(R)$ and both $v$ and $V$ are twice continuously differentiable on a bounded interval $(\alpha, \beta)$. If $V=0$ on $(-\infty, \alpha], v=0$ on $R \backslash(\alpha, \beta)$ and $-\Delta v+\frac{1}{2} r^{2} \Delta_{b} v=0$ in $(\alpha, \beta)$, then

$$
\text { (4.5) } \quad \int_{\alpha}^{\beta}\left(-\Delta V+\frac{1}{2} r^{2} \Delta_{b} v\right) v d x=v(\beta) v_{x}(\beta-0)+\frac{1}{2}\left(\frac{r}{b}\right)^{2} \int_{\beta-b}^{\beta} v(x+b) v(x) d x \text {. }
$$

Proof. Let $E$ and $\delta$ be small positive numbers. By integration by parts,

$$
\begin{aligned}
\int_{\alpha+\varepsilon}^{\beta-\delta}(-\Delta v) v d x & =-\left[v_{x} v\right]_{\alpha+\varepsilon}^{\beta-\delta}+\int_{\alpha+\varepsilon}^{\beta-\delta} v_{x} v_{x} d x \\
& =\left[v v_{x}-v_{x} v\right]_{\alpha+\varepsilon}^{\beta-\delta}+\int_{a+\varepsilon}^{\beta-\delta}(-\Delta v) v d x \\
& =\left[v v_{x}-v_{x} v\right]_{\alpha+\varepsilon}^{\beta-\delta}-\int_{a+\varepsilon}^{\beta-\delta}\left(\frac{1}{2} r^{2} \Delta_{b} v\right) v d x .
\end{aligned}
$$

Letting $\varepsilon+0$ and $\delta+0$, and noting that $v(\alpha)=v(\alpha)=v(\beta)=0$, we 
obtain

(4.6) $\quad \int_{\alpha}^{\beta}(-\Delta v) v d x=\int_{\alpha}^{\beta}\left(-\frac{1}{2} x^{2} \Delta_{b} v\right) v d x+v(\beta) v_{x}(\beta-0)$.

Noting that $v=0$ on $R \backslash(\alpha, \beta)$ and $v=0$ on $(-\infty, \alpha]$, we obtain

$$
\begin{aligned}
\int_{a}^{\beta}\left(\frac{1}{2} r^{2} \Delta_{b} v\right) v d x & =\int_{-\infty}^{\infty}\left(\frac{1}{2} r^{2} \Delta_{b} v\right) v d x=\int_{-\infty}^{\infty}\left(\frac{1}{2} r^{2} \Delta_{b} v\right) v d x \\
& =\int_{\alpha}^{\beta}\left(\frac{1}{2} r^{2} \Delta_{b} v\right) v d x+\frac{1}{2}\left(\frac{r}{b}\right)^{2} \int_{\beta}^{\beta+b} v(x-b) v(x) d x \\
& =\int_{\alpha}^{\beta}\left(\frac{1}{2} r^{2} \Delta_{b} v\right) v d x+\frac{1}{2}\left(\frac{r}{b}\right)^{2} \int_{\beta-b}^{\beta} v(x+b) v(x) d x .
\end{aligned}
$$

Now (4.5) is obtained by adding $(4.6)$ and $(4.7)$.

We here classify ss-pulses into four groups according to support and symmetry. An ss-pulse u is called a finite ss-pulse when supp[u] is compact. Finite ss-pulses are classified into two groups; one is the group of symmetric finite ss-pulses that are symmetric with respect to the center of support and the other is the group of non-symmetric ones. An ss-pulse $u$ is called an infinite ss-pulse when supp [u] = R, while $u$ is called a semi-infinite sspulse when supp[u] is a semi-infinite interval. Thus we have classified ss-pulses into four groups:

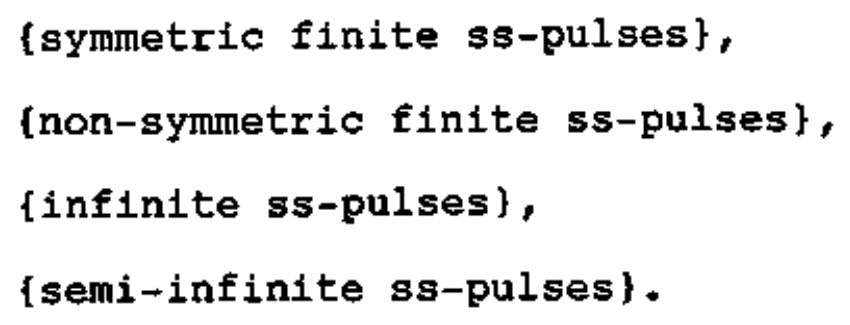

For the equation $P(2, r), I>\sqrt{2}$, we shall show the existence of a symmetric finite ss-pulse in section 5, and show the non-existence of other three kinds of ss-pulses in section 6 . 


\section{Symmetric finite standing solitary pulses}

In this section, we study the symetric finite ss-pulse of $P(2, r)$.

Let $u$ be a symmetric finite ss-pulse of $P(2, r)$. We may assume supp $[u]$ to equal an interval $\left[-\frac{s}{2}, \frac{s}{2}\right]$ (s: an unknown positive number) since any translation of $u$ also is a symmetrlc finite ss-pulse. In general, (4.1) is not equivalent to (4.2), which is obtained by differentiating (4.1). As for even functions however, (4.1) and (4.2) are mutually equivalent: if an even function is a solution of (4.2), then it also fulfils (4.1). Thus the problem to find a symmetric finite ss-pulse is described in the form of the following free boundary problem:

$$
\text { (5.1) }\left\{\begin{array}{l}
\text { Find an even function } u \in C(R) \text { and } s>0 \text { such that } \\
-\Delta u+\frac{1}{2} r^{2} \Delta u=0 \text { in }\left(-\frac{s}{2}, \frac{s}{2}\right), \\
u>0 \text { on }\left(-\frac{s}{2}, \frac{s}{2}\right), \quad u=0 \text { on } R \backslash\left(-\frac{s}{2}, \frac{s}{2}\right) .
\end{array}\right.
$$

By the coordinate transformation $x+\frac{\pi}{3} x,(5.1)$ is transformed into

$$
\text { (5.2) }\left\{\begin{array}{l}
\text { Find an even function } w \varepsilon C(R) \text { and } b>0 \text { such that } \\
-\Delta w+\frac{1}{2} x^{2} \Delta_{b} w=0 \quad \text { in }\left(-\frac{\pi}{2}, \frac{\pi}{2}\right), \\
w>0 \text { on }\left(-\frac{\pi}{2}, \frac{\pi}{2}\right), \quad w=0 \text { on } R \backslash\left(-\frac{\pi}{2}, \frac{\pi}{2}\right) .
\end{array}\right.
$$

Here is the following one-to-one correspondence between the solutions $\{u, s\}$ of $(5.1)$ and $\{w, b\}$ of $(5.2)$ :

$$
s b=\pi r \quad \text { and } w(x)=u\left(\frac{s}{\pi} x\right) \text { for } x \in R \text {. }
$$

We now consider the following eigenvalue problem: 
$E(r, b)\left\{\begin{array}{l}\text { For } r \geq 0 \text { and } b>0 \\ \text { find an even function } v \varepsilon C(R) \backslash\{0\} \text { and } \lambda E R \text { such that } \\ A_{r, b} v=\lambda v \quad \text { in }\left(-\frac{\pi}{2}, \frac{\pi}{2}\right), \quad v=0 \quad \text { on } R \backslash\left(-\frac{\pi}{2}, \frac{\pi}{2}\right),\end{array}\right.$ where $A_{r, b}$ denotes the operator $-\Delta+\frac{1}{2} r^{2} \Delta_{b}$ clearly, $\{u, s\}$ is a solution of $(5,1)$ if and only if $\left\{v(x)=u\left(\frac{s}{\pi} x\right), 0\right\}$ is a solution of $E(I, \pi r / s)$ that satisfies the positivity condition
(5.4)
$\mathrm{v}>0$
on $\left(-\frac{\pi}{2}, \frac{\pi}{2}\right)$.

In the following four subsections, we shall show that when $r>$ $\sqrt{2}, \mathrm{P}(2, \mathrm{r})$ has a symmetric finite ss-pulse characterized as a principal elgenfunction of $\mathrm{E}(\mathrm{r}, \mathrm{b})$ through the coordinate transformation $x \rightarrow \frac{\pi}{s} x$. We shall also show that the symmetric finite ss-pulse of $P(2, r)$ is unique up to the coordinate translation and the multipiication by a positive number. In practice, we shall prove the following properties of the family of eigenvalue problems $E(r, b)$, $r \geq 0$ and $b>0:$

(1) each $E(I, b)$ has an increasing sequence of eigenvalues (Subsection 5.1),

(2) for each $I>\sqrt{ } 2$, there exists a unique positive number $b(r)$ such that the principal eigenvalue of $E(r, b(r))$ equals zero (Subsectión 5.2 ),

(3) the principal eigenvalue of $E(r, b(r))$ is simple and a principal eigenfunction is positive on $\left(-\frac{\pi}{2}, \frac{\pi}{2}\right)$ or negative on $\left(-\frac{\pi}{2}, \frac{\pi}{2}\right.$ ) (Subsection 5.3 ),

(4) no $n$-th eigenfunction $(n>1)$ is of one $\operatorname{sign}$ on $\left(-\frac{\pi}{2}, \frac{\pi}{2}\right)$ when the corresponding eigenvalue equals zero (Subsection 5.3). 
5.1. Variational formulation of the eigenvalue problem

We practically consider the variational formulation of $E(r, b)$. For this purpose, we introduce a function space $\mathrm{H}_{\mathrm{s}}$ and a symetric continuous bilinear form $a_{r, b}$ in $H_{s}$ associated with the operator $A_{r, b}$. The function space $H_{g}$ is a subspace of $H^{1}(R)$ given by

$$
H_{S}=\left\{V \varepsilon H^{1}(R) ; V=0 \text { on } R \backslash\left(-\frac{\pi}{2}, \frac{\pi}{2}\right), V(x)=v(-x) \text { for } x \in R\right\}
$$

equipped with the norm

$$
\|v\|_{2,1}=\{(v, v)+(\nabla v, \nabla v)\}^{1 / 2} \text {. }
$$

The space $H_{S}$ is identified with the space that consists of even functions belonging to $\mathrm{H}_{0}^{1}\left(\left(-\frac{\pi}{2}, \frac{\pi}{2}\right)\right) \quad \mathrm{H}_{0}^{1}\left(\left(-\frac{\pi}{2}, \frac{\pi}{2}\right)\right)$ : the closure of $\mathrm{C}_{0}^{\infty}\left(\left(-\frac{\pi}{2}, \frac{\pi}{2}\right)\right)$ in $\left.H^{1}\left(\left(-\frac{\pi}{2}, \frac{\pi}{2}\right)\right)\right)$. The bilinear form $a_{r, b}: H_{s} \times H_{S}+R$ is given by

$$
a_{r, b}(v, w)=(\nabla v, \nabla w)-\frac{1}{2} x^{2}\left(\nabla_{b} v, \nabla_{b} w\right) \text {. }
$$

We thus obtain the variational formulation of $E(r, b)$ :

$e(r, b)\left\{\begin{array}{l}\text { For } r \geq 0 \text { and } b>0, \text { find } v \in H_{S} \backslash\{0\} \text { and } \lambda \in R \text { such that } \\ a_{r, b}(v, w)=\lambda(v, w) \quad \text { for all } w \in H_{s} .\end{array}\right.$

A solution of $E(r, b)$ becomes a solution of $e(r, b)$, of course. Let $\{v, \lambda\}$ be a solution of $e(r, b)$. We can identify $v$ with the solution of the equation

$$
-\Delta w=-\frac{1}{2} r^{2} \Delta_{b} v+\lambda v \quad \text { in }\left(-\frac{\pi}{2}, \frac{\pi}{2}\right), \quad w=0 \quad \text { on } R \backslash\left(-\frac{\pi}{2}, \frac{\pi}{2}\right)
$$

The solution $w$ is twice continuously differentiable on $\left(-\frac{\pi}{2}, \frac{\pi}{2}\right)$ since the right-hand side of the above equation belongs to $H^{1}(R)$. Conse- 
quently, $e(r, b)$ is equivalent to $E(r, b)$.

We associate a symmetric continuous bilinear form in $\mathrm{H}_{S}$

$$
\tilde{a}_{r, b}(v, w)=a_{r, b}(v, w)+2\left(\frac{r}{b}\right)^{2}(v, w)
$$

with $a_{r, b}$, and consider another eigenvalue problem

$\tilde{\varepsilon}(r, b)\left\{\begin{array}{l}\text { For } r \geq 0 \text { and } b>0, \text { find } v \varepsilon H_{S} \backslash\{0\} \text { and } \dot{\lambda} \varepsilon R \text { such that } \\ \tilde{a}_{r, b}(v, w)=\bar{\lambda}(v, w) \text { for all } w \varepsilon H_{S^{*}} .\end{array}\right.$

clearly, $\{v, \lambda\}$ is a solution of $e(r, b)$ if and only if $\left\{v, \lambda+2\left(\frac{r}{b}\right)^{2}\right\}$ is a solution of $\tilde{e}(r, b)$. By the estimate

$$
\left\|\nabla_{b} v\right\|=\frac{1}{b}\left\{\int_{-\infty}^{\infty}\left|v\left(x+\frac{b}{2}\right)-v\left(x-\frac{b}{2}\right)\right|^{2} d x\right\}^{1 / 2} \leq \frac{2}{b}\|v\| \text { for all } v \varepsilon H_{S}
$$

and the Poincare inequality, the bilinear form $\tilde{a}_{r, b}$ is $H_{s}$-elliptic:

$$
\vec{a}_{r, b}(v, v)=\|\nabla v\|^{2}-\frac{l}{2} r^{2}\left\|\nabla_{b} v\right\|^{2}+2\left(\frac{r}{b}\right)^{2}\|v\|^{2}
$$

$z\|\nabla v\|^{2}=\left\{2\|\nabla v\|^{2}+\pi^{2}\|\nabla v\|^{2}\right\} /\left(2+\pi^{2}\right)$

$$
\geq 2\|v\|_{2,1}^{2} /\left(2+\pi^{2}\right) \quad \text { for all } \mathrm{v} \varepsilon \mathrm{H}_{\mathrm{s}} \text {. }
$$

Proposition 5.1 (Characterization of principal eigenvalue). (1) The eigenvalue problem $e(r, b)$ has an increasing sequence of eigenvalues

$$
-\infty<\lambda_{1}(r, b) \leq \lambda_{2}(r, b) \leq \ldots \ldots \text {, with } \lim _{i \rightarrow \infty} \lambda_{i}(r, b)=\infty .
$$

(2) Eigenfunctions $v_{i}$ associated with $\lambda_{i}(i=1,2, \ldots)$ can be orthonormarized so that

$$
a_{r, b}\left(v_{i}, v_{j}\right)=\lambda_{i} \delta_{i j} \text { and }\left(v_{i}, v_{j}\right)=\delta_{i j} \text { for all } i \text { and } j,
$$

where $\delta$ denotes Kronecker's delta.

(3) The principal eigenvalue $\lambda_{1}(r, b)$ is characterized by 


$$
\lambda_{1}(r, b)=\inf \left\{R_{r, b}(v) ; v \varepsilon \mathrm{H}_{S} \backslash(0\}\right\}=R_{r, b}\left(v_{1}\right)
$$

with the aid of the Rayleigh guotient

$$
R_{r, b}(v) \equiv a_{r, b}(v, v) /\|v\|^{2} \text { for } v \varepsilon H_{g} \backslash\{0\} \text {. }
$$

(4) A function $v \varepsilon \mathrm{H}_{\mathrm{S}} \backslash\{0\}$ becomes a principal eigenfunction of $e(r, b)$ if $R_{r, b}(v)=\lambda_{\uparrow}(r, b)$.

Proof. The assertions of the proposition have been proved for the symmetric continuous $H_{s}$-elliptic bilinear forms. (For instance, Iefer to Ciarlet $[11, \mathrm{pp} .283-286]$, Riesz and Nagy $[39, \mathrm{pp} .231$ $235]$ and Kato $(23, \mathrm{pp} .260-262]$.) This together with the foregoing argument on the relation between $e(r, b)$ and $\tilde{e}(r, b)$ completes the proof. $\quad$ a

The operator $\Delta_{b}$ is reduced to $-2 / b^{2}$ when $b \geq \pi$, hence the solutions of $e(r, b), b \geq \pi$, are given by

$$
v_{i}(x)=\cos (21-1) x \text { on }\left(-\frac{\pi}{2}, \frac{\pi}{2}\right) \text { and } \quad \lambda_{i}=(2 i-1)^{2}-\left(\frac{\pi}{b}\right)^{2} \text {. }
$$

Thus, when $b \geq \pi$, the princlpal eigenfunction of $e(r, b)$ is positive on $\left(-\frac{\pi}{2}, \frac{\pi}{2}\right)$ or negative on $\left(-\frac{\pi}{2}, \frac{\pi}{2}\right)$, the support of the eigenfunction equals $\left[-\frac{\pi}{2}, \frac{\pi}{2}\right]$ and

$$
\left\|\nabla v_{i}\right\|^{2} /\left\|v_{i}\right\|^{2}=(2 i-1)^{2} \quad \text { for } i=1,2, \ldots \text {. }
$$

When $0<b<\pi$, a principal eigenfunction of e(r,b) is not always of one sign on $\left(-\frac{\pi}{2}, \frac{\pi}{2}\right)$, which will be discussed in subsection 5.3 . But we obtain the following two propositions about the support of an eigenfunction and the $\mathrm{H}_{\mathrm{s}}$-norm of a principal eigenfunction.

Proposition 5.2 (Support of an eigenfunction). The support of an eigenfunction of e(r,b) equals $\left[-\frac{\pi}{2}, \frac{\pi}{2}\right]$. 
Proof. We omit the complete proof, and show only that the support of an eigenfunction is never included in $\left(-\frac{\pi}{2}, \frac{\pi}{2}\right)$. We have already noted that the support of an eigenfunction of $e(r, b)$ equals $\left[-\frac{\pi}{2}, \frac{\pi}{2}\right]$ when $b \geq \pi$. Suppose that $e(I, b), b<\pi$, has a solution $\{v, \lambda\}$ such that supp $[v]$ is included in an interval $\left[-\frac{\pi}{2}+d, \frac{\pi}{2}-d\right]$ (d: a positive number). We may assume that $\pi / b$ is not an integer since we can replace $\{v, \lambda\}$ by some $\left\{w(x)=v(c x), c^{2} \lambda\right\} \quad(1-2 d / \pi<c<1)$ if necessary, which is a solution of $e\left(r, \frac{b}{c}\right)$ such that supp $(w]$ is included in $\left(-\frac{\pi}{2}, \frac{\pi}{2}\right)$. Let $n=\max \{i \varepsilon z ;$ ib $<\pi\}$. We may assume $d$ to be less than $\frac{1}{2}(\pi-n b)$. We partition $\left[-\frac{\pi}{2}, \frac{\pi}{2}\right]$ into the intervals

$$
\begin{cases}{\left[y_{i}, y_{i}+d\right],} & \left(y_{i}+d, z_{i}-d\right), \quad\left[z_{i}-d, z_{i}\right] \quad(i=0,1, \ldots, n), \\ \left(z_{i}, y_{i+1}\right) & (i=0,1, \ldots, n-1),\end{cases}
$$

where $y_{i}=-\frac{\pi}{2}+i b$ and $z_{i}=\frac{\pi}{2}-(n-i) b(i=0,1, \ldots, n)$. (See Figure 5.1.) since $\{v, \lambda\}$, is regarded as a solution of $E(x, b)$ and $v=0$ on $\left[\mathrm{y}_{0}, \mathrm{y}_{0}+\mathrm{d}\right]$,

$$
0=A_{r, b} v(x)-\lambda v(x)=\frac{1}{2}\left(\frac{r}{b}\right)^{2} v(x+b) \text { for } x \varepsilon\left(y_{0}, y_{0}+d\right) \text {. }
$$

This implies that $v=0$ on $\left[y_{1}, y_{1}+d\right]$ since $v \varepsilon C(R)$. By mathematical induction, we know that $v=0$ on $\left[y_{i}, y_{i}+d\right](i=0, \ldots, n)$. The fact that $v=0$ on $\left[z_{t}-d, z_{i}\right](i=0, \ldots, n)$ is shown by the same method of proof.

Let $v_{i}$ be the restriction of $v$ on $\left(y_{i}+a, z_{i}-d\right)(i=0, \ldots, n)$. We regard $v_{i}^{\prime} s$ as functions defined on the interval $(0, \pi-n b-2 d)$ through suitable coordinate translations. Since $\{v, \lambda\}$ is regarded as a solution of $E(r, b)$ and $v=0$ on $\left[y_{i}, y_{i}+d\right]$ and $\left[z_{i}-d, z_{1}\right](i=0, \ldots, n)$, $v_{i}^{\prime} s(i=0,1, \ldots, n)$ satisfy 


$$
\left\{\begin{array}{l}
-\Delta v_{i}+\frac{1}{2}\left(\frac{r}{b}\right)^{2}\left(v_{i-1}-2 v_{i}+v_{i+1}\right)=\lambda v_{i} \quad \text { in }(0, \pi-n b-2 d) \\
v_{i}(0)=\left(v_{i}\right)_{x}(0)=v_{i}(\pi-n b-2 d)=\left(v_{i}\right) x(\pi-n b-2 d)=0
\end{array}\right.
$$

where $v_{-1} \equiv v_{n+1} \equiv 0$. This system of linear equations has no nontrivial solution for any $\lambda \varepsilon R$. Hence $v=0$ on $\left[Y_{i}+d, z_{i}-d\right]$ $(i=0, \ldots, n)$. The fact that $v=0$ on $\left[z_{1}, y_{1+1}\right](i=0, \ldots n-1)$ is shown by the same method of proof. Thus,

$$
v=0 \quad \text { on }\left[-\frac{\pi}{2}, \frac{\pi}{2}\right] \text {, }
$$

which contradicts the assumption that $v$ is an eigenfunction of e $(r, b)$.

Proposition 5.3 ( $\mathrm{H}_{\mathrm{s}}$-norm of a principal eigenfunction). A principal eigenfunction $v$ of $e(r, b)$ satisfies the estimate

$$
\|\nabla v\|^{2} /\|v\|^{2} \leq 1+2\left(\frac{r}{b}\right)^{2} \equiv K(r, b) .
$$

Proof. Let $v$ be a principal eigenfunction of $e(r, b)$, associated with $\lambda_{1}(r, b)$. Then $v$ is a principal eigenfunction of $\tilde{\theta}(r, b)$, associated with $\tilde{\lambda}_{1}(r, b)=\lambda_{1}(r, b)+2\left(\frac{r}{b}\right)^{2}$. Using (5.5) and applying (3) of Proposition 5.1 to $\vec{e}(r, b)$, we obtain

$$
\begin{aligned}
\|\nabla v\|^{2} /\|v\|^{2} & \leq \tilde{a}_{r, b}(v, v) /\|v\|^{2}=\inf \left\{\tilde{a}_{r, b}(w, w) /\|w\|^{2} ; w \varepsilon H_{s} \backslash\{0\}\right\} \\
& \leq \inf \left\{\left[\|\nabla w\|^{2}+2\left(\frac{r}{b}\right)^{2}\|w\|^{2}\right] /\|w\|^{2} ; w \varepsilon H_{s} \backslash(0\}\right\} .
\end{aligned}
$$

This together with the equality

$$
\begin{aligned}
\inf \left\{\|\nabla w\|^{2} /\|w\|^{2} ; w \varepsilon H_{S} \backslash\{0\}\right\} & =\operatorname{Inf}\left\{R_{0, b}(w) ; w \varepsilon H_{s} \backslash\{0\}\right\} \\
& =\lambda_{1}(0, b)=1
\end{aligned}
$$

(an application of (3) of Proposition 5.1 to $(0, b)$ ) completes the proof. 


\subsection{Zero points of the principal eigenvalue}

The purpose of the present subsection is to prove

Theorem 5.4 (Unique existence of a zero point). Let $I>\sqrt{ } 2$. Then there exists a unlque positive number $b=b(r)$ such that the principal eigenvalue $\lambda_{1}(r, b)$ of $e(r, b)$ just equals zero. The function $b(r)$ has the following properties:

$$
\begin{aligned}
& b(r)=r \text { for } r \geq \pi \text { and } b(r)<\pi \text { for } r<\pi, \\
& b(r) \text { increases with } r \text {, } \\
& b(r) \text { is a continuous function of } r \text {, } \\
& b(r) \text { tends to zero as } r \text { tends to } \sqrt{ } \text {. }
\end{aligned}
$$

(Figure 5.2 shows the graph of $b(r)$ obtained by a finite difference approximation for $e(r, b)$. The function $b(r)$ has the property

$$
b(r)<r \text { for } r<\pi \text {, }
$$

which will be proved in the next subsection.) I

We begin with the uniqueness of $b(r)$. Let us show that if $\lambda_{1}(r, b)=\lambda_{1}(r, c)=0$ for $0<b \leq c$, then $b=c$. Let $w$ be $a$ principal eigenfunction of $e(r, c)$, assoclated with $\lambda_{1}(r, c)$. Define a function $\tilde{w}$ by $\tilde{w}(x)=w\left(\frac{C}{b} x\right)$ for $x \in R$. Then $\tilde{w} \in H_{s}$ and

$$
R_{r, b}(\bar{w})=\left(\frac{c}{b}\right)^{2} R_{r, c}(w)=\left(\frac{c}{b}\right)^{2} \lambda_{1}(r, c)=0 .
$$

Th1s together with (4) of Proposition 5.1 implies that $\tilde{w}$ is a principal eigenfunction of $\theta(x, b)$, associated with $\lambda_{1}(r, b)$. Hence $b$ should equal $c$ since if $b<c$, then supp $[\tilde{w}]$ is included in $\left(-\frac{\pi}{2}, \frac{\pi}{2}\right)$ and $\tilde{w}$ is never an eigenfunction of $e(r, b)$ by Proposition 5.2. We 
thus obtain the uniqueness of $b(r)$.

We next discuss the existence of $b(r)$. As stated in Subsection 5.1 ,

$$
\lambda_{1}(r, b)=1-\left(\frac{r}{b}\right)^{2} \quad \text { for } b \geq \pi
$$

wh1ch implies

$$
\lambda_{1}(x, b) \geq 0 \text { for } b \geq \max \{\pi, r\}
$$

on the other hand,

(5.7) $\lambda_{1}(r, b)<0$ for $0<b<B(r)$ and $r>\sqrt{2}$,

where $B(r)$ denotes the smallest positive solution of the equation

$$
1-\frac{1}{2} x^{2} \frac{\pi-x}{\pi}\left\{\frac{\sin (x / 2)}{x / 2}\right\}^{2}=0
$$

This is because the principal elgenvalue is estimated by

$$
\lambda_{1}(r, b) \leq R_{r, b}(w)<\frac{\pi}{2}\left[1-\frac{1}{2} r \frac{2 \pi-b}{\pi}\left\{\frac{\sin (b / 2)}{b / 2}\right\}^{2}\right)
$$

(13) of Proposition 5.1), where $w E H_{s}$ is given by $w(x)=\cos x$ for $|x| \leq \frac{\pi}{2}$. By $(5.6)$ and $(5.7)$, for $r>\sqrt{ } 2$, there exists a positive number $b(r)$ such that $\lambda_{1}(x, b(r))=0$ if $\lambda_{1}(r, b)$ 1s continuous with respect to $b$. Consequently, the following proposition completes the proof of the first assertion of Theorem 5.4 .

Proposition 5.5 (Continulty of the principal eigenvalue). The principal eigenvalue $\lambda_{1}(r, b)$ of $e(r, b)$ is a locally Lipschitz continuous function of $(r, b) \in(0, \infty) \times(0, \infty)$.

Proof. We fix arbitrary positive numbers $R$ and $B$. Let $p, q$, $b$ and $c$ be positive numbers such that

$$
\mathrm{p}<\mathrm{R}, \mathrm{q}<\mathrm{R} \text { and } \mathrm{B}<\mathrm{b} \leq \mathrm{c} \text {. }
$$

We first estimate $\left|\lambda_{1}(q, b)-\lambda_{1}(p, b)\right|$. Let $v_{q}$ and $v_{p}$ be principal eigenfunctions associated with $\lambda_{1}(q, b)$ and $\lambda_{1}(p, b)$, respectively. 
Using the Rayleigh quotient, wo obtain

$$
\left\{\begin{aligned}
\lambda_{1}(q, b)-\lambda_{1}(p, b) & =R_{q, b}\left(v_{q}\right)-R_{p, b}\left(v_{p}\right) \leq R_{q, b}\left(v_{p}\right)-R_{p, b}\left(v_{p}\right) \\
& =\frac{1}{2}(p+q)(p-q)\left\|\nabla_{b} v_{p}\right\|^{2} /\left\|v_{p}\right\|^{2}, \\
\lambda_{1}(p, b)-\lambda_{1}(q, b) & \leq \frac{1}{2}(p+q)(q-p)\left\|\nabla_{b} v_{q}\right\|^{2} /\left\|v_{q}\right\|^{2} .
\end{aligned}\right.
$$

From the above estimates, Lemma 4.1 and Proposition 5.3, 1t follows that

$$
\begin{aligned}
\left|\lambda_{1}(q, b)-\lambda_{1}(p, b)\right| & \leq \frac{1}{2}(p+q)|q-p| \max \left\{\left\|\nabla_{b} v_{p}\right\|^{2} /\left\|v_{p}\right\|^{2}, \mid \nabla_{b} v_{q}\left\|^{2} /\right\| v_{q} \|^{2}\right\} \\
& \leq \frac{1}{2}(p+q)|q-p| \max \left(\left\|\nabla_{p}\right\|^{2} /\left\|v_{p}\right\|^{2}, \mid \nabla v_{q}\left\|^{2} /\right\| v_{q} \|^{2}\right\} \\
& \leq \frac{1}{2}(p+q)|q-p| K(R, b) \leq R|q-p| K(R, B) .
\end{aligned}
$$

We next estimate $\left|\lambda_{1}(p, b)-\lambda_{1}(p, c)\right|$. By Lemmas 4.1 and 4.3,

$$
\|v\|^{2}\left|R_{p, b}(v)-R_{p, c}(v)\right|=\frac{1}{2} p^{2}\left|\left\|\nabla_{b} v\right\|^{2}-\left\|\nabla_{c} v\right\|^{2}\right|
$$

$$
\leq \frac{1}{2} p^{2}\left\{\left\|\nabla_{b} v\right\|+\left\|\nabla_{c} v\right\|\right\}\left\|\nabla_{b} v-\nabla_{c} v\right\| \leq \frac{2}{c} p^{2}\|\nabla v\|^{2}|c-b| \text { for all } v \in H_{s} \text {. }
$$

Let $v_{b}$ and $v_{c}$ be principal eigenfunctions associated with $\lambda_{1}(p, b)$ and $\lambda_{1}(p, c)$, respectively. By (3) of Proposition 5.1,

$$
\left\{\begin{array}{l}
\lambda_{1}(p, b)-\lambda_{1}(p, c)=R_{p, b}\left(v_{b}\right)-R_{p, c}\left(v_{c}\right) \leq R_{p, b}\left(v_{c}\right)-R_{p, c}\left(v_{c}\right) \\
\lambda_{1}(p, c)-\lambda_{1}(p, b) \leq R_{p, c}\left(v_{b}\right)-R_{p, b}\left(v_{b}\right) .
\end{array}\right.
$$

Applying (5.9) to $v=v_{c}$ and $v=v_{b}$ and using Proposition 5.3, we obtain 


$$
\begin{aligned}
\left|\lambda_{1}(p, b)-\lambda_{1}(p, c)\right| & \leq \max \left\{\left|R_{p, b}\left(v_{c}\right)-R_{p}, c\left(v_{c}\right)\right|,\left|R_{p, c}\left(v_{b}\right)-R_{p, b}\left(v_{b}\right)\right|\right\} \\
& \leq \frac{2}{c} p^{2}|c-b| \max \left\{\left\|\nabla_{c}\right\|^{2} /\left\|v_{c}\right\|^{2},\left\|v_{b}\right\|^{2} /\left\|v_{b}\right\|^{2}\right\} \\
& \leq \frac{2}{c} p^{2}|c-b| k(p, \beta) \leq \frac{2}{B} R^{2}|c-b| k(R, \beta)
\end{aligned}
$$

This together with $(5.8)$ completes the proof:

$$
\begin{aligned}
&\left|\lambda_{1}(q, b)-\lambda_{1}(p, c)\right| \leq\left|\lambda_{1}(q, b)-\lambda_{1}(p, b)\right|+\left|\lambda_{1}(p, b)-\lambda_{1}(p, c)\right| \\
& \leq R\left\{\frac{2}{\beta} R|c-b|+|q-p|\right\} K(R, B) \\
& \text { for } 0<p<R, 0<q<R \text { and } 0<B<b \leq c \text {. }
\end{aligned}
$$

We now proceed to the discussion on the properties of the function $b(r)$.

The first property follows from the uniqueness of $b(r)$ and the fact that $\lambda_{1}(r, b)=1-\left(\frac{\underline{k}}{b}\right)^{2}$ for $b \geq \pi$.

Let us show the monotonicity (2):

$$
\mathrm{b}(\mathrm{p})<\mathrm{b}(\mathrm{q}) \text { for } \sqrt{2}<\mathrm{p}<\mathrm{q} \text {. }
$$

Let $\mathrm{v}$ be a principal eigenfunction associated with $\lambda_{1}(p, b(p))$. By (3) of Proposition 5.1 and the fact that $\nabla_{b}(p) v \neq 0$,

$$
\begin{aligned}
0 & =\lambda_{1}(p, b(p))=R_{p, b(p)}(v)=\left\{\|\nabla v\|^{2}-\frac{1}{2} p^{2}\|\nabla b(p) v\|^{2}\right\} /\|v\|^{2} \\
& >\left\{\|\nabla v\|^{2}-\frac{1}{2^{q}}{ }^{2}\left\|\nabla_{b(p)} v\right\|^{2}\right\} /\|v\|^{2}=R_{q, b(p)}(v)
\end{aligned}
$$

which 1mplies that $\lambda_{1}(g, b(p))<0$. This together with $(5.6)$ and Proposition 5.5 implies that $b(p)<b(q)$.

We next show the continuity of $b(r)$. Let $\left\{r_{n}\right\}$ be an arbitrary monotone sequence of positive numbers $r_{n}>\sqrt{2}$ that converges to $r>$ $\sqrt{2}$. Since $\left\{b\left(r_{n}\right)\right\}$ becomes a bounded monotone sequence by the property $(2), b\left(r_{n}\right)$ converges to a positive number $b \geqq b\left(\right.$ inf $\left.\left\{r_{n}\right\}\right)>$ 
0. Proposition 5.5 yields

$$
\left|\lambda_{1}(r, \bar{b})\right|=\left|\lambda_{1}(r, \vec{b})-\lambda_{1}\left(r_{n}, b\left(r_{n}\right)\right)\right| \leq K\left\{\left|r-r_{n}\right|+\left|b-b\left(r_{n}\right)\right|\right\},
$$

where $\mathrm{K}$ is a positive constant independent of $\mathrm{n}$. The right-hand side of the above inequality tends to zero as $n$ tends to infinity. Hence $\lambda_{1}(r, \vec{b})$ equals zero, and $\lim _{n \rightarrow \infty} b\left(r_{n}\right)=\bar{b}=b(r)=b\left(\lim m_{n \rightarrow \infty} r_{n}\right)$ since $b(r)$ uniquely exists.

Let us show the last property. Let $\left(\mathrm{r}_{\mathrm{n}}\right)$ be an arbitrary monotone decreasing sequence of positive numbers $r_{n}>\sqrt{2}$ that converges to $\sqrt{2}$. By the property $(2)$ of $b(r), b\left(r_{n}\right)$ converges to a nonnegative number $\bar{F}$. Suppose that $\bar{B}>0$. Then, using Proposition 5.5, we obtain the estimate

$$
\begin{aligned}
\left|\lambda_{1}(\sqrt{2}, 5)\right| & =\left|\lambda_{1}(\sqrt{2}, \bar{b})-\lambda_{1}\left(r_{n}, b\left(r_{n}\right)\right)\right| \\
& \leq K\left\{\left|\sqrt{2}-r_{n}\right|+\left|\vec{b}-b\left(r_{n}\right)\right|\right\}+0 \text { as } n+\infty,
\end{aligned}
$$

where $K$ is a positive constant independent of $n$. The above estimate tmplies that $\lambda_{1}(\sqrt{2}, \bar{b})=0$, which contradicts Lemma $4+1$. Thus we have obtained the property (4). Now, the proof of Theorem 5.4 is completed except for the parenthesized assertion. 


\subsection{Sign of an eigenfunction}

In the previous subsection, we have shown that for each $I>\sqrt{ } 2$, there exists a unique positive number $b(r)$ such that the principal eigenvalue $\lambda_{1}(r, b(r))$ of $e(r, b(r))$ equals zero. The present subsection is devoted to showing

(1) a principal eigenfunction assoclated with $\lambda_{1}(r, b(r))$ is positive on $\left(-\frac{\pi}{2}, \frac{\pi}{2}\right)$ or negative on $\left(-\frac{\pi}{2}, \frac{\pi}{2}\right)$.

(2) when the n-th eigenvalue $\lambda_{n}(r, b)(n>1)$ equals zero, no eigenfunction associated with $\lambda_{n}(r, b)$ is of one sign on $\left(-\frac{\pi}{2}, \frac{\pi}{2}\right)$.

When $b \geq \pi$, a principal eigenfunction of $e(r, b)$ is positive on $\left(-\frac{\pi}{2}, \frac{\pi}{2}\right)$ or negative on $\left(-\frac{\pi}{2}, \frac{\pi}{2}\right)$ and no $n$-th eigenfunction $(n>1)$ is of one sign on the interval, as stated in subsection 5.1 . When $b<\pi$, however, this is not always true. See Figure 5.3, which depicts eigenvalues and typical patterns of eigenfunctions obtained by a finite difference approximation for $\theta(r, b)$ with a fixed $r$ and various values of $b$. The number of nodes of a principal eigenfunction $v$, which we here define by the number of zero points of $v$ on $\left[-\frac{\pi}{2}, \frac{\pi}{2}\right)$, increases with decreasing $b$, and $n$-th eigenfunctions $(n>1)$ can be of one sign on $\left(-\frac{\pi}{2}, \frac{\pi}{2}\right)$ for suitable values of $b$.

To understand the behavior of eigenvalues and eigenfunctions of $e(r, b)$, let us consider another eigenvalue problem:

$e^{a}(r, b)\left\{\begin{array}{l}\text { For } r \geq 0 \text { and } b>0, \text { find } w \in H_{a} \backslash\{0\} \text { and } \mu \varepsilon R \text { such that } \\ { }^{a} r, b(w, z)=\mu(w, z) \text { for all } z \varepsilon H_{a}\end{array}\right.$

where $\mathrm{H}_{\mathrm{a}}$ denotes the orthogonal complement of $\mathrm{H}_{\mathbf{S}}$ in the space 


$$
H=\left\{v \in H^{1}(R) ; v=0 \text { on } R \backslash\left(-\frac{\pi}{2}, \frac{\pi}{2}\right)\right\} \approx H_{0}^{1}\left(\left(-\frac{\pi}{2}, \frac{\pi}{2}\right)\right),
$$

that is,

$$
\mathrm{H}_{\mathrm{a}}=\left\{\mathrm{w} \varepsilon \mathrm{H}^{1}(\mathrm{R}) ; \mathrm{w}=0 \text { on } \mathrm{R} \backslash\left(-\frac{\pi}{2}, \frac{\pi}{2}\right), \mathrm{w}(\mathrm{x})=-\mathrm{w}(-\mathrm{x}) \text { for } \mathrm{x} \varepsilon \mathrm{R}\right\} \text {. }
$$

The same assertions as those in Proposition 5.1 hold for $e^{a}(r, b)$.

Figure 5.4 depicts eigenvalues $\mu_{n}$ and typical patterns of eigenfunctions $w_{n}$ obtained by a finjte difference approximation for $e^{a}(r, b)$. See Figure 5.5, which is obtained by putting together Figures 5.3 and 5.4. Figure 5.5 reveals that the curves of $n$-th elgenvalues $(n=1,2, \ldots)$ of $e(r, \cdot)$ and $e^{a}(r, \cdot)$ intersect with each other many times, and that the number of nodes of an n-th eigenfunction of $e(r, *)$ increases at points b such that

$\lambda_{n}(r, b+y)<\mu_{n}(r, b+y)$ and $\lambda_{n}(r, b-y)>\mu_{n}(r, b-y)$ for $0<y<\varepsilon$, where $\varepsilon$ is a small positive number. At points $(r, b)$ such that $\lambda_{n}(r, b)=\mu_{n}(r, b)$, there is a relation

$$
w_{n}=\left(v_{n}\right)_{x}
$$

between the eigenfunctions $v_{n}$ and $w_{n}$ of $e(r, b)$ and $e^{a}(r, b)$, respectively.

The behavior of elgenvalues and eigenfunctions of $e(r, b)$ is complicated as observed in the above. Nevertheless, the behavior of an eigenfunction is simple on the region where the corresponding eigenvalue is non-negative, and we can arrive at the following two theorems, Theorems 5.6 and 5.8, that imply the properties (1) and (2) described in the beginning of the present subsection.

Theorem 5.6 (Sign of a principal eigenfunction). If the principal eigenvalue $\lambda_{1}(r, b)$ of $e(r, b)$ is non-negative, then a principal eigenfunction $v$ associated with $\lambda_{1}(r, b)$ is positive on $\left(-\frac{\pi}{2}, \frac{\pi}{2}\right)$ or negative on $\left(-\frac{\pi}{2}, \frac{\pi}{2}\right)$. More precisely, if $\lambda_{1}(r, b) \geq 0$, then 
$v>0$ on $\left(-\frac{\pi}{2}, \frac{\pi}{2}\right)$ and $v 1$ s non-decreasing on $(-\infty, 0)$

or

$v<0$ on $\left(-\frac{\pi}{2}, \frac{\pi}{2}\right)$ and $v$ is non-increasing on $(-\infty, 0)$.

Proof. Let $\mathrm{v}$ be a principal eigenfunction associated with a non-negative principal elgenvalue $\lambda_{1}(r, b)$ of $e(r, b)$. If $v$ is nondecreasing (resp, non-increasing) on $(-\infty, 0)$, then $v(x)>0$ (resp. $v(x)<0)$ for $x \in\left(-\frac{\pi}{2}, \frac{\pi}{2}\right)$ by Proposition 5.2. Hence it suffices for the proof to show that $v$ is un1modal: $v$ is non-decreasing on $(-\infty, 0)$ or $v$ is non-increasing on $(-\infty, 0)$.

We begin by showing that a unimodal function $w \varepsilon H_{s}$ given by

$$
\left\{\begin{array}{l}
w(x)=\int_{-\pi / 2}^{x}\left|v_{y}(y)\right| d y \text { for } x \leq 0, \\
w(x)=w(-x) \text { for } x>0
\end{array}\right.
$$

is a principal eigenfunction associated with $\lambda_{1}(r, b)$. clearly, (5.10) $\|\nabla w\|=\|\nabla v\|$.

It is easy to show that $w \geq|v|$ on $R$ and $\left|\nabla_{b} w\right| \geq\left|\nabla_{b} v\right|$ on $R$, which Imply $\mid w\|\geq\| v \|$ and $\left\|\nabla_{b} w\right\| \geq \| \nabla_{b} v \mid$, respectively. By (3) of Proposition 5.1, the assumption on the sign of $\lambda_{1}(r, b),(5.10)$ and $(5.12)$,

$$
\begin{aligned}
\lambda_{1}(r, b) & =R_{r, b}(v)=\left\{\|\nabla v\|^{2}-\frac{1}{2} r^{2}\left\|\nabla_{b} v\right\|^{2}\right\} /\|v\|^{2} \\
& \geq\left\{\left\|\nabla_{v}\right\|^{2}-\frac{1}{2} r^{2} \|\left.\nabla_{b} v\right|^{2}\right\} /\|w\|^{2} \geq\left\{\|\nabla w\|^{2}-\frac{1}{2} r^{2}\left\|\nabla_{b} w\right\|^{2}\right\} /\|w\|^{2} \\
& =R_{r, b}(w) .
\end{aligned}
$$

Hence $w$ is a principal eigenfunction by (3) and (4) of Proposition 5.1 . 
Now, suppose that $v$ is not unimodal. Then $v_{x}$ is not of one sign on $\left(-\frac{\pi}{2}, 0\right)$. Fix a small positive number $d$ such that $\frac{\pi}{2}+d<0$ and $-\frac{\pi}{2}-b+d<-\frac{\pi}{2}$. We first deal with the case where $v_{x}$ is not of one sign on $\left(-\frac{\pi}{2},-\frac{\pi}{2}+d\right)$. In this case,

$$
\begin{aligned}
\left|\nabla_{b} v\left(-\frac{\pi}{2}-\frac{b}{2}+d\right)\right| & =\frac{1}{b}\left|v\left(-\frac{\pi}{2}+d\right)-v\left(-\frac{\pi}{2}-b+d\right)\right|=\frac{1}{b}\left|v\left(-\frac{\pi}{2}+d\right)-v\left(-\frac{\pi}{2}\right)\right| \\
= & \frac{1}{b}\left|\int_{-\pi / 2}^{-\pi / 2+d} v_{x}(x) d x\right|<\frac{1}{b} \int_{-\pi / 2}^{-\pi / 2+d}\left|v_{x}(x)\right| d x=\left|\nabla_{b} w\left(-\frac{\pi}{2}-\frac{b}{2}+d\right)\right| .
\end{aligned}
$$

This together with (5.11) 1mplies that $\left\|\nabla_{b} w>\right\| \nabla_{b} v \|$ since both $\nabla_{b} w$ and $\nabla_{\mathrm{b}} \mathrm{v}$ are continuous. Hence $(5.13)$ is replaced by

$$
\lambda_{1}(r, b)=R_{r, b}(v)>R_{r, b}(w),
$$

which contradicts (3) of Proposition 5.1. We next deal with the other case where $v_{x}$ is of one sign on $\left(-\frac{\pi}{2},-\frac{\pi}{2}+d\right)$. We may assume that $v_{x} \geq 0$ on $\left(-\frac{\pi}{2},-\frac{\pi}{2}+d\right)$. Then, $w-v=0$ on $\left(-\frac{\pi}{2},-\frac{\pi}{2}+d\right)$, while $w(0)-v(0)=\int_{-\pi / 2}^{0}\left\{|v x(x)|-v_{x}(x)\right\} d y>0$ since $v_{x}$ is not of one sign on $\left(-\frac{\pi}{2}, 0\right)$. Hence $w-v$ is a principal elgenfunction whose support is included in $\left[-\frac{\pi}{2}+d, \frac{\pi}{2}-d\right]$. This contradicts Proposition 5.2. I

Corollary 5.7 (Simplicity of the princtpal eigenvalue). The principal eigenvalue of $e(r, b)$ is simple when $1 t$ is non-negative.

Proof. Suppose that a non-negative principal eigenvalue $\lambda_{1}(r, b)$ of $e(r, b)$ is not simple. Then, by (2) of Proposition 5.1, there exist two principal eigenfunctions $v_{1}$ and $v_{2}$ associated with $\lambda_{1}(r, b)=\lambda_{2}(r, b) \geq 0$ such that $\left(v_{1}, v_{2}\right)=0$. This contradicts Theorem 5.6. I 
Theorem 5.8 (Sign of an n-th eigenfunction). When the n-th eigenvalue $\lambda_{n}(r, b)$ of $e(r, b)$ is non-negative ( $\left.n>1\right)$, no eigenfunction associated with $\lambda_{n}(r, b)$ is of one sign on the interval $\left(-\frac{\pi}{2}, \frac{\pi}{2}\right)$.

Proof. Let $\mathrm{n}$ be an arbitrary integer greater than the unity. When $b \geq b(r)$, the principal eigenvalue of $e(r, b)$ is non-negative by the uniqueness of $b(r)$, and $e(r, b)$ has a principal eigenfunction $\tilde{v}$ such that $\tilde{v}>0$ on $\left(\frac{\pi}{2}, \frac{\pi}{2}\right)$ by Theorem 5.6 . Hence, when $b \geq b(r)$, no $n$-th elgenfunction $v_{n}$ of $e(r, b)$ is of one sign on $\left(-\frac{\pi}{2}, \frac{\pi}{2}\right)$ since $\left(\tilde{v}, v_{n}\right)=0$ by (2) of Proposition 5.1. Suppose that there exists a positive number $b<b(r)$ such that $\lambda_{n}(r, b) \geqq 0$ and an $n-t h$ eigenfunction $v_{n}$ assoclated with $\lambda_{n}(r, b)$ is of one $\operatorname{sign}$ on $\left(-\frac{\pi}{2}, \frac{\pi}{2}\right)$ :

$$
A_{r, b} v_{n}=\lambda_{n} v_{n} \text { in }\left(-\frac{\pi}{2}, \frac{\pi}{2}\right), \quad v_{n} \geq 0 \text { on }\left(-\frac{\pi}{2}, \frac{\pi}{2}\right) \text { and } \lambda_{n} \geq 0 \text {. }
$$

We may assume that $\left(v_{n}, 1\right)=2$. Let $\tilde{v}$ be a principal eigenfunction associated with $\lambda_{1}(r, b(r))$ :

$$
A_{r, b(r)} \bar{v}=0 \quad \text { in }\left(-\frac{\pi}{2}, \frac{\pi}{2}\right) \text {. }
$$

The function $v(x)=\bar{v}\left(\frac{b(r) x}{b}\right)$ satisfies

$$
\text { (5.t5) } \quad A_{r, b^{V}}=0 \quad \text { in }\left(-\frac{d}{2}, \frac{d}{2}\right) \text {, }
$$

where $d=\pi b / b(r)$. We may assume that $(v, 1)=2$ by Theorem 5.6 . With $v_{n}$ and $v$, we associate non-decreasing functions

$$
v_{n}(x)=\int_{0}^{x} v_{n}(y) d y \text { and } v(x)=\int_{0}^{x} v(y) d y \text {, }
$$

respectively. Integrating $(5.14)$ and (5.15), we obtain

$$
\mathrm{A}_{\mathrm{r}, \mathrm{b}} \mathrm{V}_{\mathrm{n}}=\lambda_{\mathrm{n}} \mathrm{V}_{\mathrm{n}} \text { in }\left(-\frac{\pi}{2}, \frac{\pi}{2}\right) \text { and } \mathrm{A}_{\mathrm{r}, \mathrm{b}} \mathrm{V}=0 \text { in }\left(-\frac{\mathrm{d}}{2}, \frac{\mathrm{d}}{2}\right) \text {, }
$$


respectively, since $v_{n}$ and $v$ are even functions. Hence $w_{n}(x)=$ $1+v_{n}\left(x-\frac{\pi}{2}\right)$ and $w(x)=1+V\left(x-\frac{d}{2}\right)$ satisfy

(5.16) $A_{r, b} W_{n}=\lambda_{n} W_{n}-\lambda_{n}$ in $(0, \pi)$ and $A_{r, b} W=0$ in $(0, d)$, respectively, and

(5.17) $w_{n}=w=0$ on $(-\infty, 0], w_{n}=2$ on $[\pi, \infty)$ and $w=2$ on $[d, \infty)$. Let $w(x)=v\left(x-\frac{d}{2}\right)$. Then, since $v$ satisfles $(5.15), w$ satisfies

$$
{ }^{A}, b^{w}=0 \text { in }(0, d) \text {, }
$$

and $w=0$ on $R \backslash(0, d)$. Applying Lemma 4.4 to $\{w, w\}$, we obtain

$$
0=\int_{0}^{d}\left(A, b_{r}\right) w d x=2 w_{x}(d-0)+\left(\frac{r}{b}\right)^{2} \int_{d-b}^{d} w(x) d x
$$

by the second equation in $(5.16)$ and $(5.17)$. We next apply Lemma 4.4 to $\left\{w, w_{n}\right)$. This results in

$$
\int_{0}^{d}\left(A, b w_{n}\right) w d x=w_{n}(d) w_{x}(d-0)+\frac{1}{2}\left(\frac{r}{b}\right)^{2} \int_{d-b}^{d} w_{n}(x+b) w(x) d x
$$

$$
>\frac{1}{2} w_{n}(d)\left\{2 w_{x}(d-0)+\left(\frac{r}{b}\right)^{2} \int_{d-b}^{d} w(x) d x\right\}=0
$$

since $d=\pi b / b(r)<\pi$ and $w_{n}$ is strictly increasing on $(0, \pi)$ by the assumption on the sign of $v_{n}$ and Proposition 5.2. On the other hand, noting the symmetry of with respect to $x=\frac{a}{2}$ and the antisymetry of $w_{n}-1$ with respect to $x=\frac{\pi}{2}$, we obtain

$$
\begin{aligned}
\int_{0}^{d}\left(\lambda_{n} w_{n}-\lambda_{n}\right) w d x & =\lambda_{n}\left\{\int_{0}^{d / 2}\left(w_{n}-1\right) w d x+\int_{d / 2}^{d}\left(w_{n}-1\right) w d x\right\} \\
& =\lambda_{n} \int_{0}^{d / 2}\left\{w_{n}(x)+w_{n}(d-x)-2\right\} w(x) d x \\
& \leqq \lambda_{n} \int_{0}^{d / 2}\left(w_{n}(x)+w_{n}(\pi-x)-2\right\} w(x) d x=0
\end{aligned}
$$

since $\lambda_{n} \geq 0, d<\pi$ and $w_{n}$ is strictly increasing on $(0, \pi)$. This and $(5.18)$ contradict the first equation in (5.16). I 
Using Theorem 5.6, we can prove (5.19) $b(r)<r$ for $r<\pi$

(Theorem 5.4). Let $v$ be a principal eigenfunction associated with $\lambda_{1}(r, b(r)), r<\pi$, We may assume $v$ to be positive on $\left(-\frac{\pi}{2}, \frac{\pi}{2}\right)$ by Theorem 5.6. Then, since $b(x)<\pi$ by Theorem 5.4,

$$
\int_{-\infty}^{\infty}(v(x+b(r))+v(x-b(r))) v(x) d x>0
$$

By (3) of Proposition 5.1 and (5.20),

$$
\begin{aligned}
0 & =\|v\|^{2} R_{r, b(r)}(v) \\
& =\|\nabla v\|^{2}+\frac{1}{2}(r / b(r))^{2} \int_{-\infty}^{\infty}(v(x+b(r))+v(x-b(r))-2 v(x)) v(x) d x \\
& >\|\nabla v\|^{2}-(r / b(r))^{2} \int_{-\infty}^{\infty} v(x)^{2} d x=\|\nabla v\|^{2}-(r / b(r))^{2}\|v\|^{2},
\end{aligned}
$$

which implies $(5,19)$ since $\|\nabla w\| \geq\|w\|$ for all $w \varepsilon H_{S^{\prime}}$ as shown in the proof of Proposition 5.3. 


\subsection{Existence and uniqueness}

We now prove

Theorem 5.9 (Symmetric finite ss-pulses of $P(2, r)$ for $r>\sqrt{ } 2$ ). Let $r>\sqrt{2}$. Then $P(2, r)$ has a unique symmetric finite ss-pulse $u_{r}$ such that

(5.21) $u_{r}$ is an even function and $u_{r}(0)=1$.

Moreover, a function $u$ is a symmetric finite ss-pulse of $P(2, r)$ if and only if $u$ is expressed in the form

$$
u(x)=a u_{r}(x-c) \text { for } x \in R
$$

with a positive number $a$ and a real number c. The length $s(r)$ of supp $\left[u_{I}\right]$ has the properties (1) to (4) stated in Theorem 1.1, and the symmetric finite ss-pulse $u_{r}$ has the properties (i) to (iii) stated in Theorem 1.1 .

Proof. By Theorem 5.4, for each $I>\sqrt{ } 2$, there exists a unique positive number $b(r)$ such that the principal eigenvalue of $e(r, b(r))$ equals zero. Theorem 5.6 and Corollary 5.7 assure that $e(r, b(r))$ has a unique principal eigenfunction $v_{r}$ such that $v_{r}(0)=1$. (See Figure 5.6). The principal eigenfunction $v_{r}$ is unimodal and $v_{r}>0$ on $\left(-\frac{\pi}{2}, \frac{\pi}{2}\right)$ by Theorem 5.6 .

We define a function $u_{r}$ by

$$
u_{r}(x)=v_{r}\left(\frac{b(r) x}{r}\right) \quad \text { for } x \in R \text {. }
$$

We can regard $\left\{v_{r}, 0\right\}$ as a solution of $g(r, b(r))$, which satisfies the positivity condition (5.4). Hence, by the argument in the beginning of this section, $\left\{u_{r}, \pi r / b(r)\right\}$ is a solution of $(5.1)$ and $u_{r}$ becomes a symmetric finite ss-pulse of $P(2, r)$ that satisfies (5.21). 
Suppose that $P(2, r)$ has another symmetric finite ss-pulse $u$ that satisfies (5.21). Let $s$ be the length of supp[u]. Then, by the argument in the beginning of this section, $\{u, s\}$ is a solution of (5.1) and the function $v(x)=u\left(\frac{s}{\pi}\right)$ becomes an eigenfunction of $E(r, \pi r / s)$, associated with a zero eigenvalue. Since $v$ is positive on $\left(-\frac{\pi}{2}, \frac{\pi}{2}\right)$, v should be a principal eigenfunction of $E(r, \pi r / s)$ by Theorem 5.8, and $\pi r / s=b(r)$ by Theorem 5.4. Now, by Corollary 5.7 and the condition $v(0)=u(0)=1, v=v_{r}$ and $u=u_{I^{*}}$ Thus we complete the proof of the unique existence of $\mathrm{u}_{\mathrm{r}}$.

since $m=2,(4.1)$ is linear, and it is clear that a function given by $(5.22)$ is a symmetric finite ss-pulse.

Let $u$ be an arbitrary symmetric finite ss-pulse, and denote by c the center of supp[u]. We associate a function w given by

$$
w(x)=u(x+c) / u(c) \text { for } x \in R
$$

with $u$. Then becomes a symmetric finite ss-pulse that satisfies (5.21). Hence $w=u_{r}$ by the unique existence of $u_{r}$, and

$$
u(x)=u(c) w(x-c)=u(c) u_{r}(x-c) \text { for } x \in R \text {. }
$$

The length $s(r)$ of supp $\left[u_{r}\right]$ equals $m / b(r)$ by $(5,23)$. Hence the properties (1) to (4) of $s(r)$ follow from those of $b(x)$ stated in Theorem 5.4 and $(5.19)$.

Before the proof of the properties of $u_{r}$, we show

$$
\left(u_{I}\right)\left(-\frac{1}{2} s(r)+0\right)=\frac{1}{2} \int_{S(r) / 2-r}^{s(r) / 2} u_{r}(x) d x>0 \quad \text { for } r>\sqrt{ } \text {. }
$$

The symmetric finite ss-pulse $u_{r}$ satisfies

$$
A_{r, r} u_{r} \equiv-\Delta u_{r}+\frac{1}{2} r^{2} \Delta_{r} u_{r}=0 \quad \text { in }\left(-\frac{1}{2} s(r), \frac{1}{2} s(r)\right) \text {, }
$$

and the function $U_{r}(x)=\int_{-\infty}^{x} u_{r}(y) d y$ also gatisfies 


$$
A_{r, r^{U}}=0 \quad \text { in }\left(-\frac{1}{2} s(r), \frac{1}{2} s(x)\right)
$$

since $u_{r}$ satisfies (4.1) with $m=2$. An application of Lemma 4.4 to $\left\{u_{r}, u_{r}\right\}$ yields

$$
\begin{aligned}
0 & =\int_{-s(r) / 2}^{s(r) / 2}\left(A_{r, r} U_{r}\right) u_{r} d x \\
& =U_{r}\left(\frac{1}{2} s(r)\right)\left(u_{r}\right)_{x}\left(\frac{1}{2} s(r)-0\right)+\frac{1}{2} \int_{s(r) / 2-r}^{s(r) / 2} U_{r}(x+r) u_{r}(x) d x \\
& =-\left\|u_{r}\right\|_{1}\left(u_{r}\right)_{x}\left(-\frac{1}{2} s(r)+0\right)+\frac{1}{2}\left\|u_{r}\right\|_{r} \int_{s}^{s(r) / 2} / 2-r u_{r}(x) d x,
\end{aligned}
$$

which implies (5.24). The property (i) of $u_{r}$ follows from the fact that $v_{I}$ is unimodal (Theorem 5.6). The second property is obtained by noting that

$$
v_{r}(x)=\cos x \text { on }\left[-\frac{\pi}{2}, \frac{\pi}{2}\right] \text { and } b(r)=r \text { for } r z \pi
$$

(Subsections 5.1 and 5.2). For $I>\sqrt{ }$, $u_{r}$ is twice continuously differentiable on $\left(-\frac{1}{2} s(r), \frac{1}{2} s(r)\right)$ by Proposition 2.1 . Let $\sqrt{2}<r<$ $\pi$. Then, the point $x_{-}=-\frac{1}{2} s(I)+r$ belongs to $\left(-\frac{1}{2} s(r), \frac{1}{2} s(r)\right)$ by the property (t) of $s(r)$, and

$$
\left(\Delta_{r} u_{r}\right)(x)=\frac{1}{2} r^{2}\left(\Delta_{r} u_{r}\right)(x)=\frac{1}{2}\left\{u_{r}(x+r)-2 u_{r}(x)+u_{r}(x-r)\right\}
$$

is not differentiable at $x_{\text {- }}$ by $(5.24)$. Hence $u_{I}$ is not thrice continuously aifferentiable on $\left(-\frac{1}{2} s(r), \frac{1}{2} s(r)\right)$ when $\sqrt{2}<r<\pi$. 
6. Non-existence of other kinds of standing solitary pulses

We show in this section that the equation $P(2, r)$ has no other kind of ss-pulse but the symmetric finite sa-pulse.

Non-existence of a non-symmetric finite ss-pulse. Suppose that $u$ is a non-symmetric finite ss-pulse of $P(2, r)$. We may assume that $\operatorname{supp}[u]=\left[-\frac{s}{2}, \frac{s}{2}\right]$ ( $s:$ a positive number). We define an even function $u_{s}$ by

$$
u_{s}(x)=\frac{1}{2}\{u(x)+u(-x)\} \text { for } x \in R \text {. }
$$

since $u$ is an ss-pulse, $u_{s}>0$ on $\left(-\frac{s}{2}, \frac{s}{2}\right)$ and $u_{s}$ satisfies

$$
-\Delta u_{s}+\frac{1}{2} r^{2} \Delta_{r} u_{s}=0 \quad \text { in }\left(-\frac{s}{2}, \frac{s}{2}\right) \text {. }
$$

Hence $u_{s}$ is a symmetric finite ss-pulse of $P(2, r)$, and $s=s(r)=$ $\pi r / b(r)$ and $u_{s}(x)=u_{s}(0) u_{r}(x)$ by Theorem 5.9 , where $u_{r}$ is the unique symmetric finite ss-pulse of $P(2, r)$ that satisfies $(5,21)$. The function $v_{s}(x)=u_{s}\left(\frac{s}{\pi} x\right)$ becomes a principal eigenfunction of $e(r, \pi r / s)$, associated with $\lambda_{j}(r, \pi r / s)=0$, as shown in the proof of Theorem $5.9(\pi r / s=b(r))$. We define an odd function $u_{a}$ and an even function $\mathrm{U}_{\mathrm{a}}$ by

$$
u_{a}(x)=\frac{1}{2}\{u(x)-u(-x)\} \text { and } u_{a}(x)=\int_{-\infty}^{x} u_{a}(y) d y \text { for } x \in R \text {. }
$$

Then, since $u$ is a non-symmetric finite ss-pulse, $u_{a} \neq 0, u_{a} \neq 0$ and $U_{a}(x)=0$ for $x \in R \backslash\left(-\frac{s}{2}, \frac{s}{2}\right)$. Moreover, $U_{a}$ satisfies

$$
-\Delta U_{a}+\frac{1}{2} r^{2} \Delta_{r} U_{a}=0 \quad \text { in }\left(-\frac{s}{2}, \frac{s}{2}\right)
$$

since $u_{a}(x)=u(x)-u_{s}(x)$ satisfies $(4.1)$ with $m=2$ in $\left(-\frac{s}{2}, \frac{s}{2}\right)$. 
Hence $v_{a}(x)=v_{a}\left(\frac{s}{\pi} x\right)$ also is a principal eigenfunction associated with $\lambda_{1}(r, \pi r / s)$. By $(5,24)$ and the definition of $v_{a}$,

$$
\begin{aligned}
& \left(v_{a}\right)_{x}\left(-\frac{\pi}{2}+0\right)=\frac{s}{\pi}\left(u_{s}\right)_{x}\left(-\frac{s}{2}+0\right)=\frac{s}{\pi} u_{s}(0)\left(u_{x}\right)\left(-\frac{s}{2}+0\right)>0, \\
& \left(v_{a}\right)_{x}\left(-\frac{\pi}{2}+0\right)=\frac{s}{\pi}\left(u_{a}\right)_{x}\left(-\frac{s}{2}+0\right)=\frac{s}{\pi} u_{a}\left(-\frac{s}{2}\right)=0,
\end{aligned}
$$

which implies that $v_{s}$ and $v_{a}$ are linearly independent. This contradicts the fact that $\lambda_{1}(r, \pi r / s)$ is simple (Corollary 5.7 ).

Non-existence of an infinite ss-pulse. An infinite ss-pulse u of $P(2, r)$ must satisfy

$$
-\Delta u+\frac{1}{2} r^{2} \Delta_{r} u=0 \text { in } R .
$$

Through the Fourier transformation, the above equation is transformed into '

$$
\left(\xi^{2}-1+\cos r \xi\right) \tilde{u}(\xi)=0,
$$

where $\bar{i}$ denotes the Fourter transformation of $u$. The function

$$
\xi^{2}-1+\cos r \xi
$$

of $\xi$ vanishes at most at a finite number of points on $R$, therefore, $\vec{u} \equiv 0$ and $u \equiv 0$. This means that $P(2, r)$ has no infinite ss-pulse.

Non-existence of a semi-infintte ss-pulse. Suppose that $P(2, r), r>\sqrt{ } 2$, has a semi-infinite ss-pulse $w$. We may assume supp $[w]$ to equal $[0, \infty]$ without loss of generality. By Theorem 5.9, $P(2, r)$ has a unique symmetric finite ss-pulse $u$ such that $(u, 1)=2$ and $\operatorname{supp}[u]=[0, s](s=s(r)=\pi r / b(r))$. We define non-decreasing functions $U$ and $w$ by

$$
v(x)=\int_{0}^{x} u(y) d y \text { and } w(x)=\int_{0}^{x} w(y) d y .
$$

Then $U$ and $w$ satisfy

$$
A_{r, I} U=0 \text { in }(0, s) \text { and } A_{r, r} W=0 \text { in }(0, \infty) \text {, }
$$


respectively, since $u$ and $w$ satisfy (4.1) with $m=2$. Apply1ng Lemma 4.4 to $\{u, v\}$ and $\{u, w\}$, Iespectively, we obtain

$$
\left\{\begin{aligned}
0=\int_{0}^{s}\left(A, r, r^{U}\right\} u d x & =2 u_{x}(s-0)+\int_{s-r}^{s} u(x) d x, \\
0=\int_{0}^{s}\left\{A_{r}, r^{W}\right\} u d x & =w(s) u_{x}(s-0)+\frac{1}{2} \int_{s-r}^{s} W(x+r) u(x) d x \\
& >\frac{1}{2} W(s)\left\{2 u_{x}(s-0)+\int_{s-r}^{s} u(x) d x\right\}
\end{aligned}\right.
$$

since $W(x)$ is strictly increasing on $(0, \infty)$. The second inequality in the above contradicts the first equality. Hence $P(2, r)$ has no semi-infinite ss-pulse. 


\section{Stationary solutions of $P(2, r)$}

In the present section, we complete the proof of Theorem 1.1 by combining the theorems obtalned in the previous sections.

Proof of Theorem 1.1. The first assertion is the same as that of Theorem 4.2 .

A stationary soiution of $P(2, r)$ that gatisfies $(1.4)$ is a symmetric finite ss-pulse of $P(2, r)$ that gatisfies $(5.21)$. Hence Theorem 5.9 implies the unique existence of $u_{r}$, the properties (1) to (4) of $s(r)$ and the properties (i) to (iii) of $u_{r}$.

Let $u$ be a function given by $(1.5)$. Then $u$ belongs to $L^{\infty}(R)$ and $L^{1}(R)$ since

$$
\left\{\begin{array}{l}
\|u\|_{\infty}=\left\|u_{r}\right\|_{\infty} \sup _{i \in \Lambda}\left\{a_{i}\right\} \leq\left\|u_{r}\right\|_{\infty} \sum_{i \in \Lambda} a_{i}<\infty, \\
\|u\|_{1} \leq\left\|u_{I}\right\|_{1} \Sigma_{i \varepsilon \Lambda_{i}}<\infty
\end{array}\right.
$$

by $(1.6)$. For each $i \varepsilon \Lambda$, let

$$
u_{i}(x)=a_{i} u_{r}\left(x-c_{i}\right) \text { for } x \in R \text {. }
$$

Theorem 5.9 assures that each $u_{1}$ is a symmetric finite ss-pulse of $P(2, r)$. Since $\operatorname{dis}\left(\operatorname{aupp}\left[u_{i}\right], \operatorname{supp}\left[u_{j}\right]\right) \geq I$ for all $i \varepsilon \Lambda, j \varepsilon \Lambda$, $i \neq j$ by (1.7), $F[u]=\sum_{i \in \Lambda} F\left[u_{i}\right]=0((2.2)$ in Proposition 2.1$)$. Hence $u$ is a stationary solution of $P(2, r)$.

Let $u$ be an arbitrary non-trivial stationary solution of $\mathrm{P}(2, \mathbf{r})$. By Theorem $3.1, \mathrm{u}$ is decomposed into

$$
u(x)=\sum_{i \varepsilon \Lambda} u_{i}(x) \text {, }
$$

where $\left\{u_{i}\right\}_{i \varepsilon \Lambda}(\Lambda$ : an index set) is a fintte or countable sequence of ss-pulses such that

(7.1) $\operatorname{dis}\left(\operatorname{supp}\left[u_{i}\right], \operatorname{supp}\left[u_{j}\right]\right) \geq r$ for all $i \varepsilon \Lambda, j \varepsilon \Lambda, i \neq j$. 
The equation $P(2, r)$ has no other kind of ss-pulse but the symmetric finite ss-pulse (Section 6). Hence each $u_{i} 1 s$ a symetric finite ss-pulse, and by Theorem 5.9, $u_{i}$ is expressed in the form

$$
u_{i}(x)=u_{i}\left(c_{i}\right) u_{r}\left(x-c_{i}\right) \text {, }
$$

where $c_{i}$ is the center of supp $\left[u_{1}\right]$. Now $u$ is expressed in the form (7.2)

$$
u(x)=\sum_{i \varepsilon \Lambda} u_{i}\left(c_{1}\right) u_{x}\left(x-c_{i}\right) \text {. }
$$

Let us show how the sequences $\left\{u_{i}\left(c_{i}\right)\right\}_{i \varepsilon \Omega}$ and $\left\{c_{i}\right\}_{i_{E} \Lambda}$ satisfy the conditions $(1.6)$ and $(1.7)$, respectively. Since each $u_{i}$ is an sspulse and

$$
\infty>\left\|u_{1}\right\|_{1}=\left\|u_{r}\right\|_{1} \sum_{i \varepsilon \Lambda} u_{i}\left(c_{i}\right)
$$

by (7.1) and (7.2), $\left\{u_{i}\left(c_{i}\right)\right\}_{i \varepsilon \Lambda}$ satisfies $(1.6)$. By (7.1) and (7.2),

$$
\begin{aligned}
\left|c_{i}-c_{j}\right|=\operatorname{dis}( & \left.\operatorname{supp}\left[u_{i}\right], \operatorname{supp}\left[u_{j}\right]\right) \\
& +\frac{1}{2}\left(\text { the length of } \operatorname{supp}\left[u_{i}\right]\right) \\
& +\frac{1}{2}\left(\text { the length of } \operatorname{supp}\left[u_{j}\right]\right)
\end{aligned}
$$

which implies $(1.7)$. We thus complete the proof of Theorem 1.1 . 


\section{Stationary solutions of $P(m, r)$ for $m \neq 2$}

The present section discusses the stationary solution of $P(m, r)$ for $m \neq 2$.

We could prove Theorem 1.1 by fully utilizing the speciality of the case $m=2$ when the equation (4.2) is reduced to the linear differential-difference equation when $m=2$. We have not yet obtained a satisfactory result for the general case of $m \neq 2$. However, we can show a partial result and clarify the difference between the cases of $m=2$ and $m \neq 2$.

The results obtained in sections 2 and 3 are valid for all $\mathrm{m}>$ 1, hence we have only to study the ss-pulse.

We first review the case of $r=\infty$. A stationary solution of $P(m, \infty)$ is always an ss-pulse (Remark 3.3). Mimura and Yamaguti [29] have shown that for each positive number $c, P(m, \infty)$ has a symmetric finite ss-pulse $u_{c}$ such that $\left\|u_{c}\right\|_{1}=c$, and that $u_{c}{ }^{*}$ is unique up to the coordinate translation.

Let $u$ be an ss-pulse of $P(m, \infty)$ such that

$$
u=0 \text { on }(-\infty, 0] \text { and } u>0 \text { on }(0, \varepsilon)
$$

where $\varepsilon$ is a small positive number, and define a non-decreasing function $U$ by $(3.1)$. When $I=\infty,(3.5)$ is reduced to

$$
J[U](x)=u_{x}(x)^{m}-U(x)\left\{\|u\|_{1}-U(x)\right)=0 \quad \text { on } R
$$

since $U(-\infty)=0$ and $U(\infty)=\|u\|_{1}$. Hence $U(x)$ is determined by the relation

$$
x=\int_{0}^{U(x)}\left\{\xi\left(\|u\|_{1}-\xi\right)\right\}^{-1 / m} d \xi \quad \text { for } x>0 .
$$


and $u(x)$ is obtained by differentiating $v(x)$. The length $s(u)$ of supp [u] $\equiv \operatorname{supp}\left[U_{x}\right]$ is given by

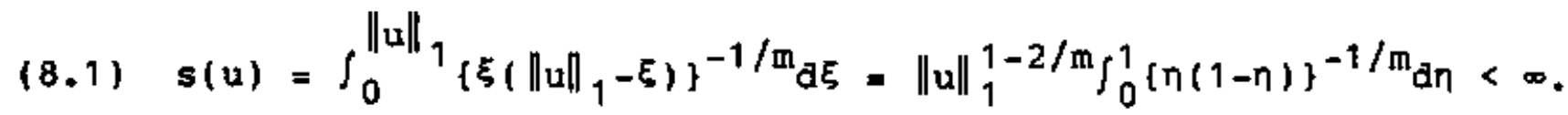
Consequently, s(u) is independent of $\|u\|_{1}$ when $m=2$, however, $s(u)$ increases with $\|u\|_{1}$ when $m>2$, while $s(u)$ decreases with increasing $\|u\|_{1}$ when $t<m<2$. This is a difference between the cases of $m=$ 2 and $m \neq 2$.

Combining $(3.7)$ and $(B .1)$, we can rewrite the above fact as

Proposition 8.1 (Support of an ss-pulse of $P(m, \infty)$ ). Let $u$ be an ss-pulse of $P(m, \infty)$. The length $g(u)$ of $\operatorname{supp}[u]$ is given by

$$
s(u)^{2}=F(m)^{2}\|u\|_{\infty}^{m-2}
$$

where $F(m)$ is the same as that given in Theorem 1.2. Hence, $s(u)$ is independent of $\|u\|_{\infty}$ when $m=2$, however, $s(u)$ increases with $\|u\|_{\infty}$ when $\mathrm{m}>2$, while $\mathrm{s}(\mathrm{u})$ decreases with increasing $\|\mathrm{u}\|_{\infty}$ when $1<\mathrm{m}<$ 2. 1

We now return to the case of $0<r<\infty$. By the definition of a stationary solution, we obtain

Proposition 8.2 (Transformation of an $s s-p u l s e$ of $P(m, r)$ for $m \neq 2)$. Assume $u$ to be an ss-pulse of $P(m, r)$ for $m \neq 2$. Then, for all $\sigma>0$,

$$
u_{\sigma}(x) \equiv \sigma^{2 /(m-2)} u(x / \sigma)
$$

for $\mathbf{x} \varepsilon \mathbf{R}$

becomes an ss-pulse of $P\left(m, \sigma_{r}\right)$. Moreover,

$$
(\sigma r)^{2}\left\|u_{\sigma}\right\|_{\infty}^{2-m}=r^{2}\|u\|_{\infty}^{2-m}
$$

for all $\sigma>0$,

$$
(\sigma r)^{m}\left\|u_{\sigma}\right\|_{.1}^{2-m}=r^{m}\|u\|_{1}^{2-m}
$$

for all o>0. 
Observing Proposition 8.2, we infer

(8.6)

"there exists a positive number $Q(m)$ such that

(a) $P(m, r)$ has no ss-pulse $u$ such that $0<r^{2}\|u\|_{\infty}^{2-m} \leq Q(m)$,

(b) for each $g>Q(m), P(m, r)$ has an ss-pulse u such that $\mathrm{r}^{2}\|\mathrm{u}\|_{\infty}^{2-\mathrm{m}}=\mathrm{q}^{n}$,

which is true for $m=2$ as stated in Theorem 1.1 .

We have not yet proved $(8.6)$ in the general case. However, we obtain Theorem 1.2 .

Proof of Theorem 1.2. Let u be a non-trivial stationary solution of $\mathrm{P}(\mathrm{m}, \mathrm{r})$ and define a non-decreasing function $\mathrm{U}$ by (3.1). Let $x=\left\{x \in R ; u(x)=\|u\|_{\alpha}\right\}$. We first consider the case where $x$ is not empty. Fix a point $x_{0} \in x$, and let

$$
x=\inf \left\{y \leq x_{0} ; z \varepsilon x \text { for all } z \in\left[y, x_{0}\right]\right\} \text {. }
$$

Then $x>-\infty$ since $u \in L^{1}(R)$, and $\int_{y}^{x} u(z) d z<(x-y)\|u\|_{\infty}$ for $y<x$. Noting that $J[U]=0$ and $u=U_{x} \geq 0$ on $\mathbf{R}$, we obtain

$$
\|u\|_{\infty}^{m}=u(x)^{m}=u_{x}(x)^{m}=\int_{x}^{x+r} u(y)(u(x)-U(y-r)) d y
$$

$$
\begin{aligned}
& \leq\|u\|_{\infty} \int_{x}^{x+r}(u(x)-u(y-r)) d y=\|u\|_{\infty} \int_{x}^{x+r} \int_{y-r}^{x} u(z) d z d y \\
& <\|u\|_{\infty} \int_{x}^{x+r}(x-y+r)\|u\|_{\infty} d y=\frac{1}{2} r^{2}\|u\|_{\infty}^{2} .
\end{aligned}
$$

In the other case where $x$ is empty, we also obtain the same estimate as (8.7). The first assertion of Theorem 1.2 follows from (8.7). Let $q$ be a positive number that satisfies $(1.9)$, and let $s=$ $r F(m) / V q \leq I . \quad$ Combining $(8.1)$ and the argument on the stationary solution of $P(m, \infty)$, we know that $P(m, \infty)$ has a symmetric finite ss- 
pulse $u_{s}$ such that the length of supp $\left[u_{s}\right]$ equals $s$. Let us show that $u_{s}$ is an ss-pulse of $P(m, r)$ satisfying $(1.10)$. The ss-pulse $u_{s}$ of $P(m, \infty)$ satisfies $(4,1)$ :

$$
\left.\frac{m}{m-1}\left(u_{s}^{m-1}\right)_{x}+\left\{\int_{-\infty}^{x} u_{s} d y-\int_{x}^{\infty} u_{s} d y\right]=0 \text { in the interior of supp [ } u_{s}\right]
$$

We can replace the second term on the left-hand side of the above equation by $\left\{\int_{x-r}^{x} u_{s} d y-\int_{x}^{x+r} u_{s} d y\right\}$ since $r z s=$ (the length of supp $\left.\left[u_{s}\right]\right)$. Then the resulting equation implies that $u_{s}$ is an sspulse of $P(m, r)$. Since $u_{s}$ is an ss-pulse of $P(m, \infty)$, it follows from $(8.2)$ that

$$
r^{2}\left\|u_{s}\right\|_{\infty}^{2-m}=\left(\frac{r}{s}\right)^{2} F(m)^{2}=q \text {, }
$$

which implies $(1.10)$. Thus the proof is completed. 0

We may appropriately conclude the present paper with the following conjecture.

\section{Conjecture (Stationary solutions of $P(m, r)$ for $\mathbb{m} \neq 2$ ).}

$$
\begin{aligned}
& P(m, r) \text { has no non-trivial stationary solution } u \text { such that } \\
& \qquad r^{2}\|u\|_{\infty}^{2-m} \leq 2 .
\end{aligned}
$$

(II) For each real number $g \geq F(m)^{2}, P(m, r)$ has an ss-pulse $u$ such that

$$
\mathrm{I}^{2}\|\mathrm{u}\|_{\infty}^{2-\mathrm{m}}=\mathrm{q}
$$

and the length of supp[u] is less than or equal to $r$. The ss-pulse $\mathrm{u}$ is unique up to the coordinate translation.

(III) For each real number $q$ such that $2<\mathrm{g}<F(m)^{2}, P(m, r)$ has an ss-pulse u such that

$$
r^{2}\|u\|_{\infty}^{2-m}=q
$$


and the length of supplu] is greater than $r$. The ss-pulse $u$ is unique up to the coordinate translation. (See Figure 8.1.) I

Acknowledgements. I am deeply grateful to Prof. M. Yamaguti of kyoto University for his encouragement and valuable advice. I also express my sincere thanks to Prof. M. Mimura and Dr. T. Nagal of Hiroshima University and Prof. H. Fujil of Kyoto Sangyo University for their continuous discussions and encouragement. 


\section{References}

[1] W. Alt, Degenerate diffusion equations with drift functionals mođelling aggregation (preprint).

[2] W. Alt, Models for mutual attraction and aggregation of motile individuals (preprint).

[3] D. G. Aronson, Regularity properties of flows through porous media, sIAM J. Appl. Math., 17(1969), 461-467.

[4] D. G. Aronson, Regularity properties of flows through porous media: A counterexample, SIAM J. Appl. Math., 19(1970), 299307.

[5] D. G. Aronson, Regularity properties of flows through porous media: The interface, Arch. Rational Mech. Anal., 37(1970), 110.

(6] D.G. Aronson, Density dependent interaction-diffusion systems, Dynamics and Modelling of Reactive systems (eds. W. E. Stewart, W. H. Ray and C. C. Conley), Academic Press, 1980.

[7] G. I. Barenblatt, On some unsteady motions of a liquid and a gas in porous medium. Prikl. Mat. Mech., 16(1952), 67-78.

[8] J. Bear, Dynamics of Fluids in Porous Media, American Elsevier, 1972 .

[9] L. A. Caffarelli and A. Friedman, Continuity of the density of a gas flow in a porous medium, Trans. Amer. Math. Soc., $252(1979), 99-113$.

[10] L. A. Caffarelli and A. Friedman, Regularity of the free boundary for the one-dimensional flow of gas in a porous medium, 
Amer. J. Math., 101 (1979), 1193-1218.

[11] P. G, Ciarlet, The Finite Element Method for Elliptic Problems, North-Holland, 1978.

[12] E. DiBenedetto and D. Hoff, An interface tracking algorithm for the porous medium equation (to appear in Trans. Amer. Math. Soc.).

[13\} B. H. Gilding, Properties of solutions of an equation in the theory of infiltration, Arch. Rational Mech, Anal., 65(1977), 203-225.

[14] B. H. Gilding and L. A. Peletier, The Cauchy problem for an equation in the theory of infiltration, Arch. Rational Mech. Anal., $61(1976), 127-140$.

[15] J. L. Graveleau and P. Jamet, A finite difference approach to some degenerate nonlinear parabolic equations, SIAM J. Appl. Math., 20(1971), 199-223.

[16] W. S. C. Gurney and R. M. Nisbet, The regulation of inhomogeneous populations, J. Theoret. Biol., 52(1975), 441-457.

[17] M. E. Gurtin and R. C. Maccany, On the diffusion of biological populations, Math. Biosci., 33(1979), 35-49.

[18] J. K. Hale, Functional Differential Equations, Springer-Verlag, 1971 .

[19] W. D. Hamilton, Geometry for the selfish herd, J. Theoret. Biol., 31(1971), 295-311.

[20] D. Hoff, A linearly lmplicit finite difference scheme for the one-dimensional porous medium equation (preprint).

[21] T. Ikeda, Discrete asymptotic behavior for a nonlinear degenerate diffusion equation (to appear in Proc. 6th Internat. Conf. on Computing Methods in Appl. Sci. and Engrg., INRIA, 1983 ). 
[22] A. S. Kalashnikov, On the occurrence of singularities in the solutions of the equation of non-stationary filtration, $\not$. vytisl. Mat. i Mat. Fiz, 7(1967), 440-444.

[23] T. Kato, Perturbation Theory for Linear Operators, SpringerVerlag, 1966.

[24] E. F. Keller and I. A. Segel, Model for chemotaxis, J. Theoret. Biol., 30(19.71), 225-234.

[25] B. F. Knerr, The porous medium equation in one dimension, Trans. Amer. Math. Soc., 234(1977), 381-415.

[26] Y. Kuramoto, Rhythms and turbulence in populations of chemical oscillator, Physica A $106(1981), 128-143$.

[27] C. G. Lange and R. M. Miura, Singular perturbation analysis of boundary-value problems for differential-difference equations, SIAM J. Appl. Math*, 42(1982), 502-531.

[28] M. Mimura, T. Nakak1 and K. Tomoeda, A numerical approach to Interface curves for some nonlinear diffusion equations, Japan J. Appl. Math. 1(1984).

[29] M. Mimura and M. Yamaguti, Pattern formation in interacting and diffusing systems in population biology, Adv. Biophys., $15(1982), 19-65$.

[30] T. Munakata, Liquid instability and freezing-Reductive perturbation approach-, J. Phys. Soc. Japan, 43(1977), 1723-1728.

[31] T. Nagai, Some nonlinear aegenerate diffusion equations with a nonlocally convective term in ecology, Hiroshima Math. J., $13(1983), 165-202$.

[32] T. Nagai and M. Mimura, Some nonlinear degenerate diffusion equations related to population dynamics, J. Math. Soc. Japan, $35(1983), 539-562$. 
[33] T. Nagai and M. Mimura, Asymptotic behavior for a nonlinear degenerate diffusion equation in population dynamics, SIAM J. Appl. Math., 43(1983), 449-464.

[34] T. Nagai and M. Mimura, Asymptotic behavior of the interface to a nonlinear degenerate diffusion equation in population dynamics (preprint).

[35] W. I. Newman, Some exact solutions to a non-linear diffusion problem in population genetics and combustion, J. Theoret. Biol., 85(1980), 325-334.

[36] A. Okubo, Diffusion and Ecological Problems: Mathematicál Models, Biomathematics 10, Springer-Verlag, 1980.

[37] O. A. Oleinik, A. S. Kalashnikov and Chzou Yui-lin, The Cauchy problem and boundary value problems for equations of the type of nonstationary filtration, Izv. Akad. Nauk., 22(1958), 667704.

[38] R. E. Pattle, Diffusion from an instantaneous point source with concentration-dependent coefficient, Quart. J. Mech. Appl. Math., 12(1959), 407-409.

[39] F. Riesz and B. Sz. Nagy, Functional Analysis, Frederic Ungar Publishing, 1955.

[40] A. E. Scheidegger, The physics of flow through porous media, University of Tronto press, 1974 .

[41] L. Schwartz, Théorie des distributıon, Hermann, 1966.

[42] R. P. Sperb, on a mathematical model describing aggregation of amoebae, Bull. Math. Biol. 41(1979), 555-571.

[43] K. Tomoeda and M. Mimura, Numertcal approximations to interface curves for a porous media equation, Hiroshima Math. J., $13(1983), 273-294$. 
Figure captions.

Figure 1.1. A pulse-like stationary solution of $P(2, r)\left(d_{1} \mathbf{r}\right.$ and $\mathrm{d}_{2} \geq \mathbf{r}$ ).

Figure 1.2. A solution of the Cauchy problem $P(2, \infty)$ obtained by a finite difference method.

Figure 5.1. A partition of the interval $\left[-\frac{\pi}{2}, \frac{\pi}{2}\right]$ (n $\left.=2\right)$.

Figure 5.2. The graph of the function $b(x)$.

Figure 5.3. Eigenvalues $\lambda$ and typical patterns of eigenfunctions of $e(r, b)(r=3)$.

Figure 5.4. Eigenvalues $\mu$ and typical patterns of eigenfunctions of $e^{a}(r, b)(r=3)$.

Figure 5.5. Elgenvalues $\lambda$ and typical patterns of eigenfunctions of $e(r, b)$ and eigenvalues $p$ of $e^{a}(r, b)(r=3)$.

Figure 5.6. Principal eigenfunctions $v_{r}$ of $e(r, b(r))(r=\pi, 2.0$, 1.5 and 1.42$)$.

Figure 8.1. Existence and non-existence of $s s-p u l s e$ of $P(m, r)$ $(s(u)=($ the length of $\operatorname{supp}[u]))$. 


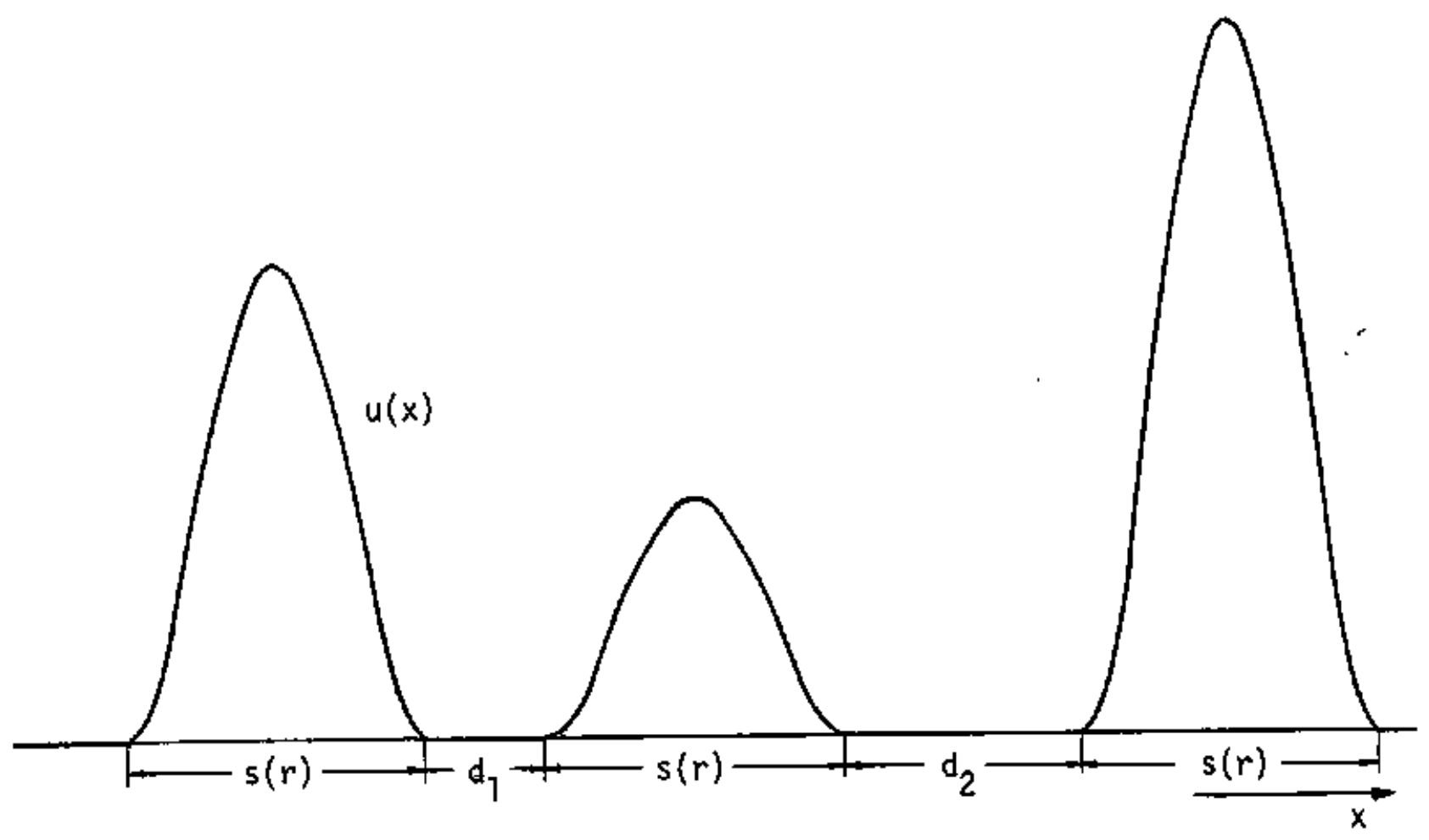

(Figure 1.1) 


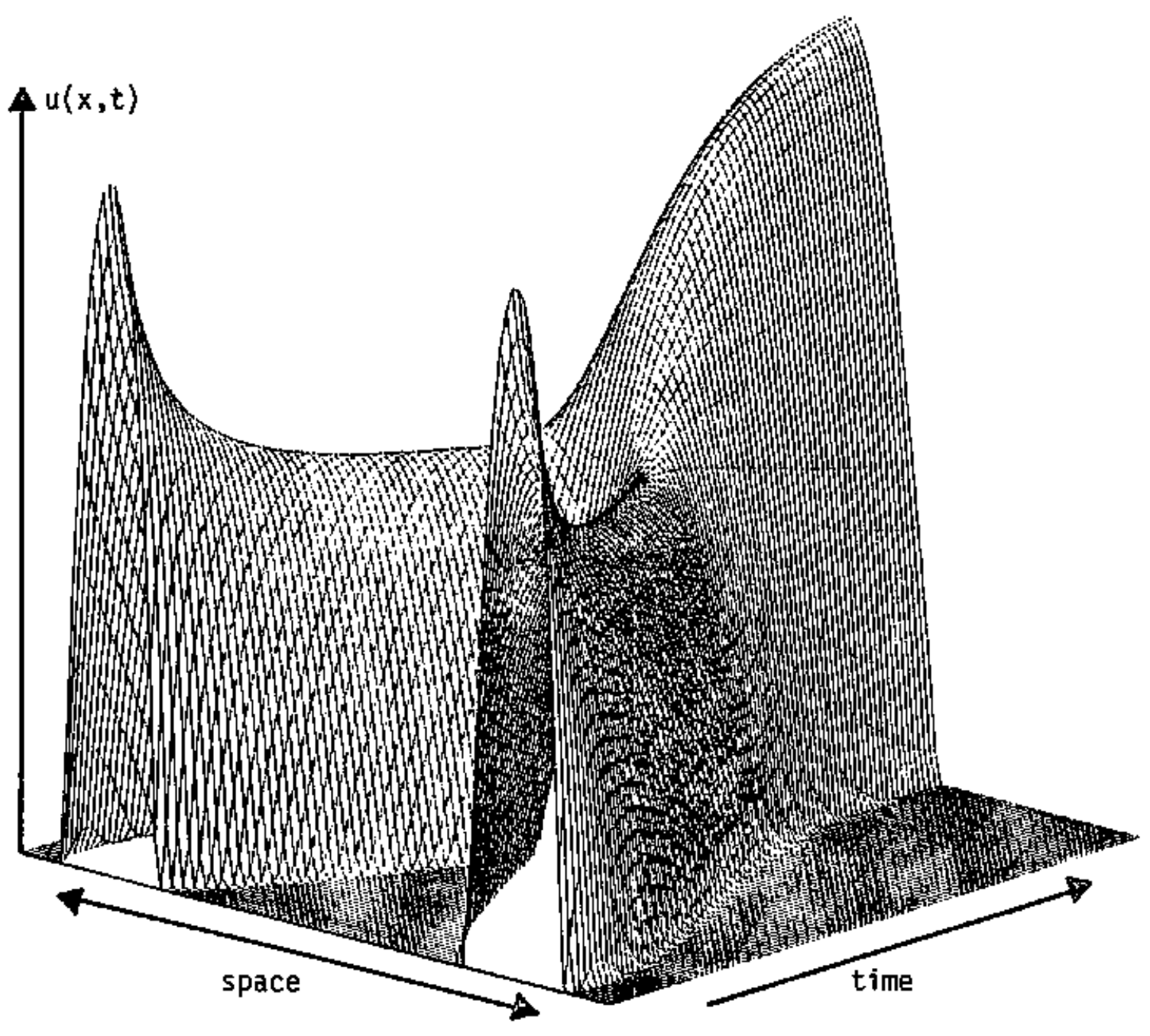

(Figure 1.2) 


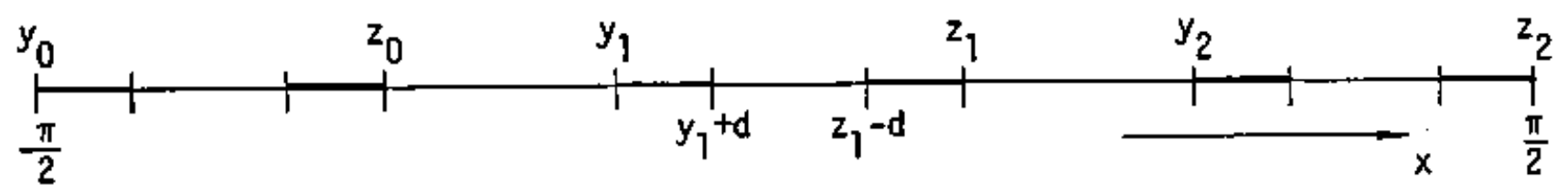

(Figure 5.1)

F-3 


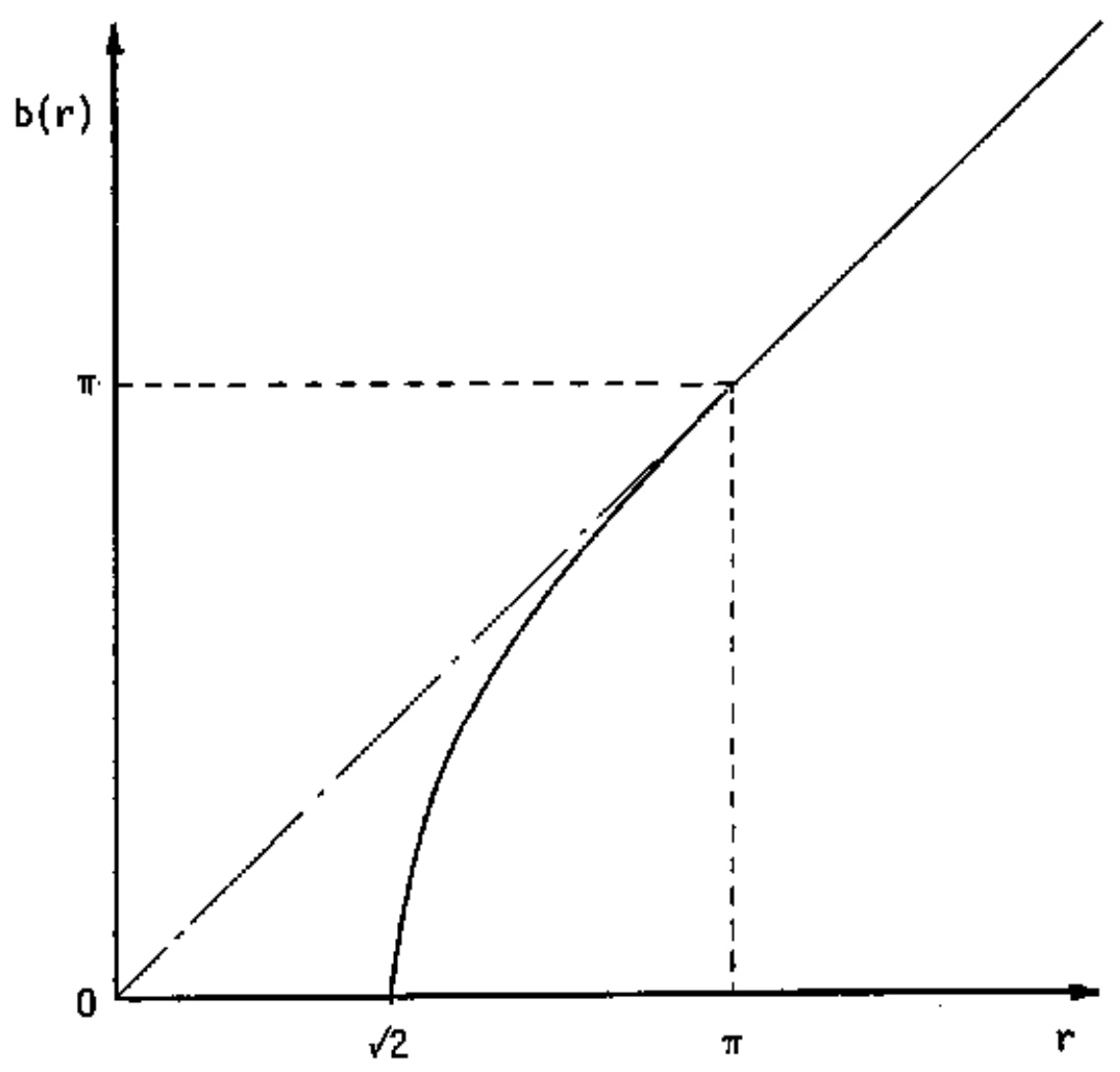

(Figure 5.2) 


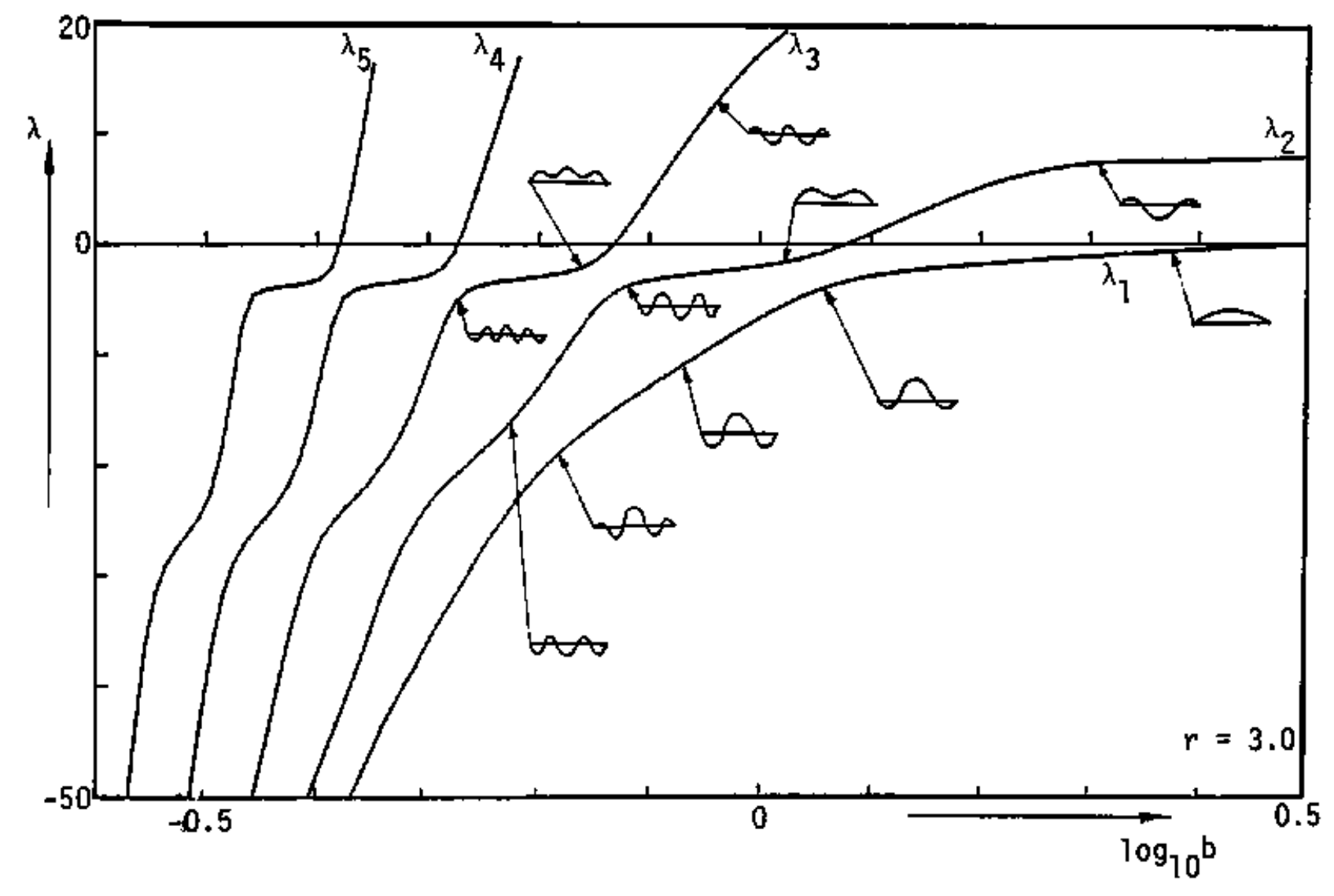

(Figure 5.3)

F-5 


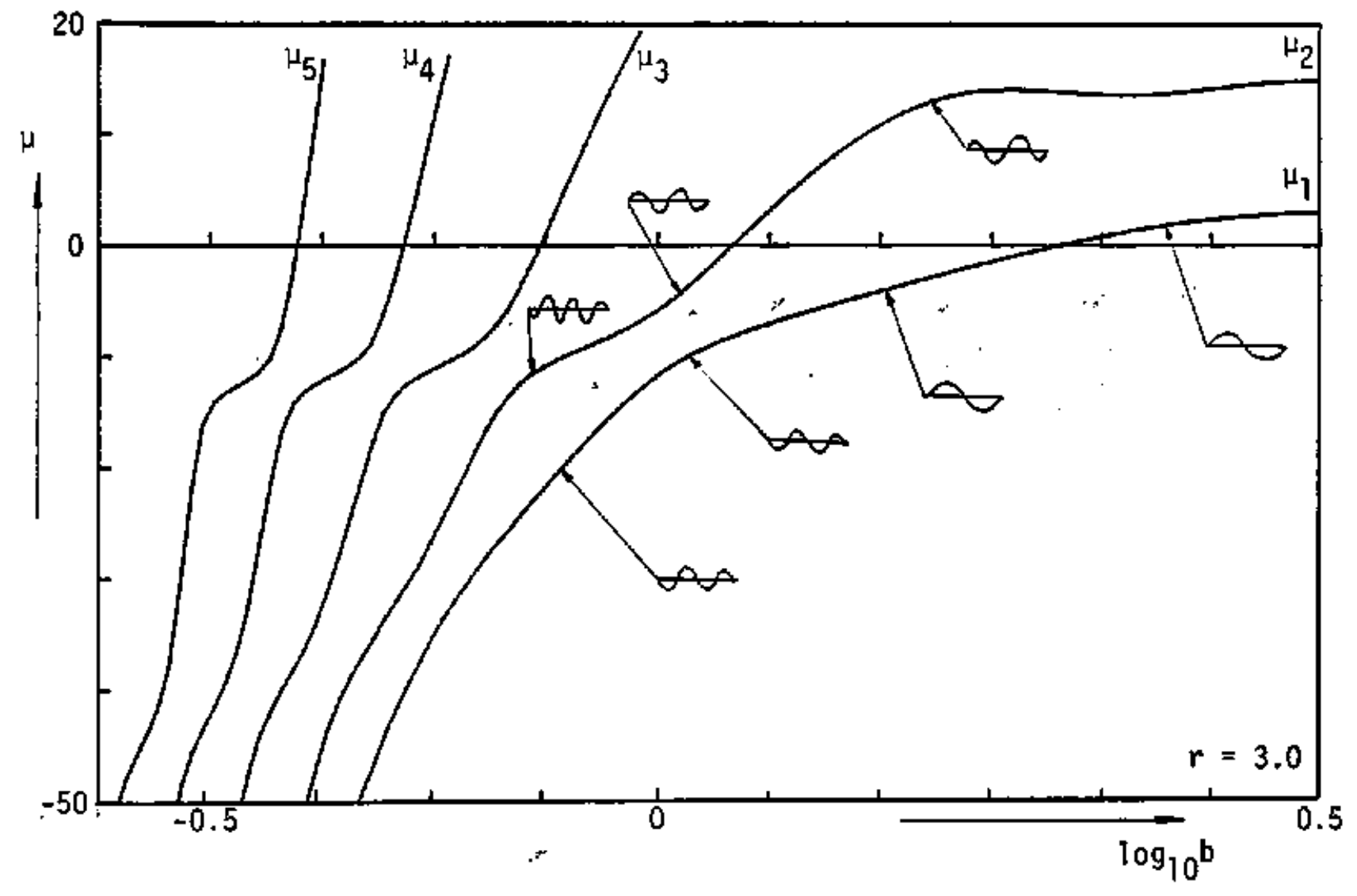

(Figure 5.4) 


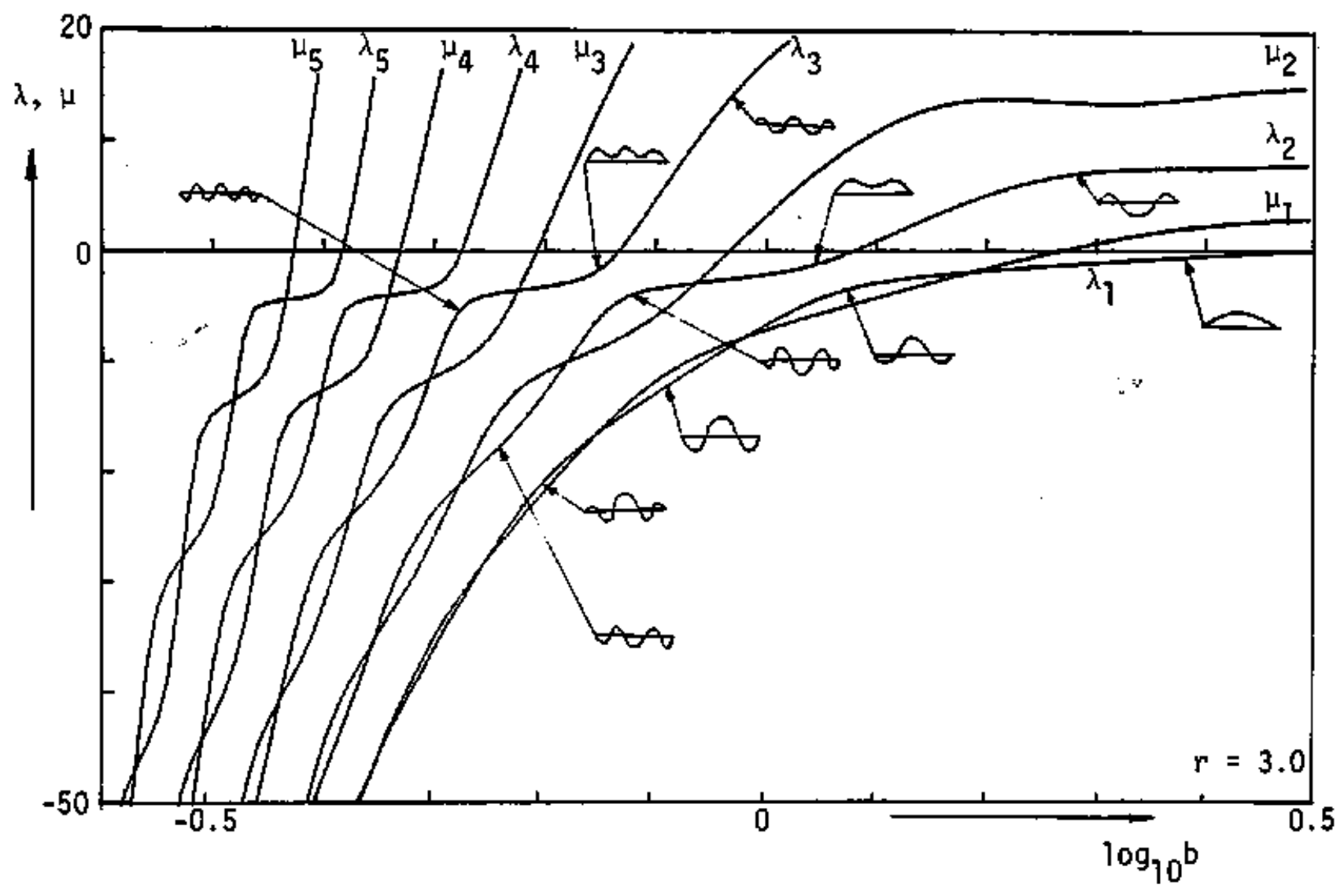

(Figure 5.5) 


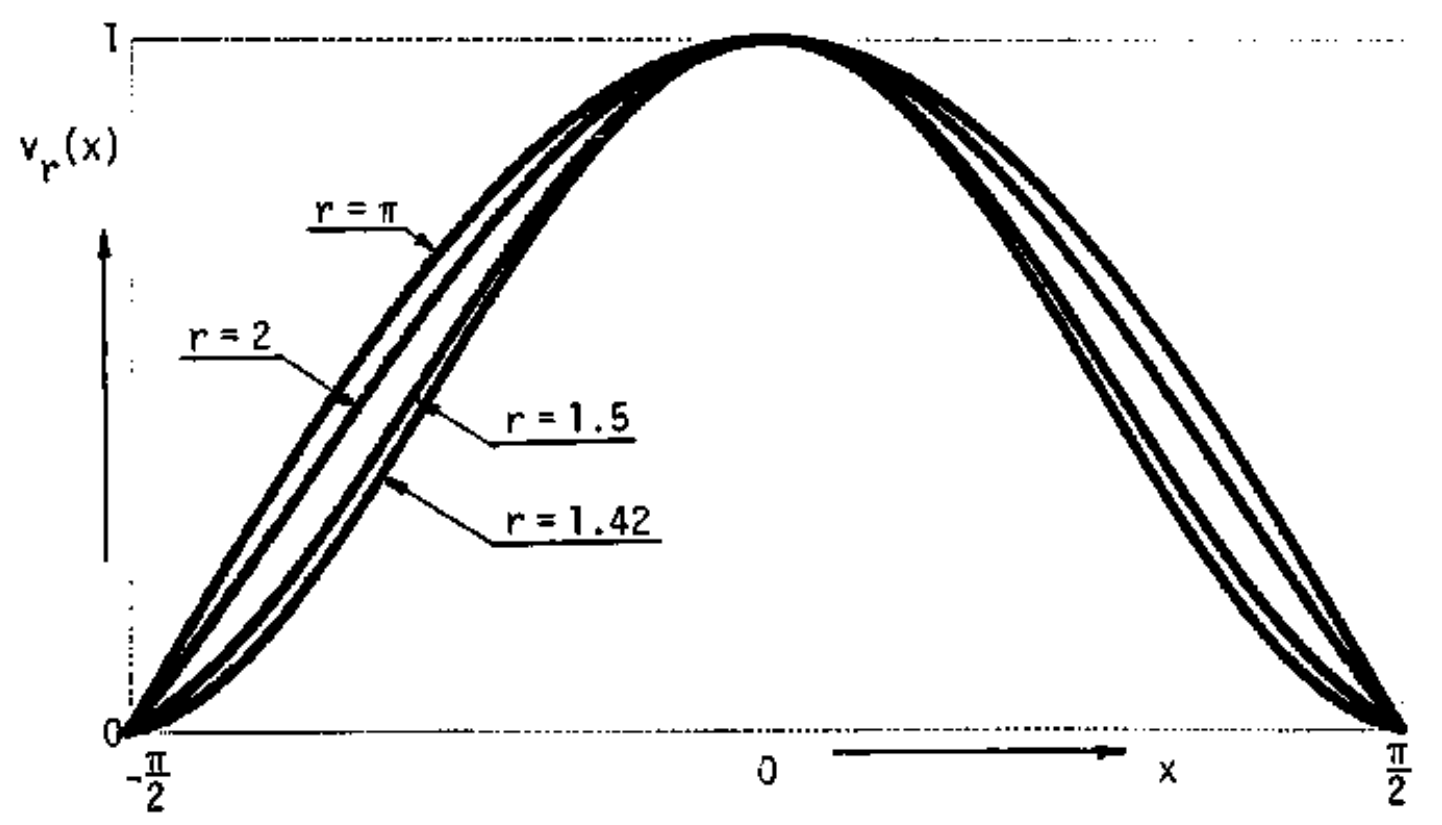

(Figure 5.6) 


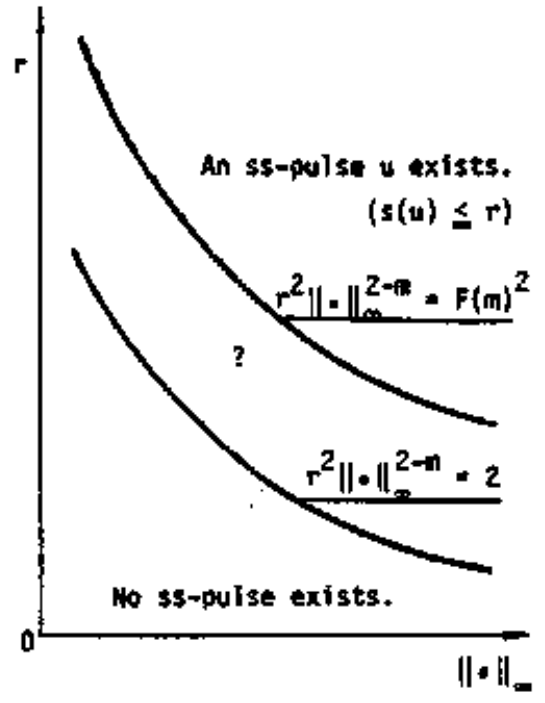

(t) $1<m<2$

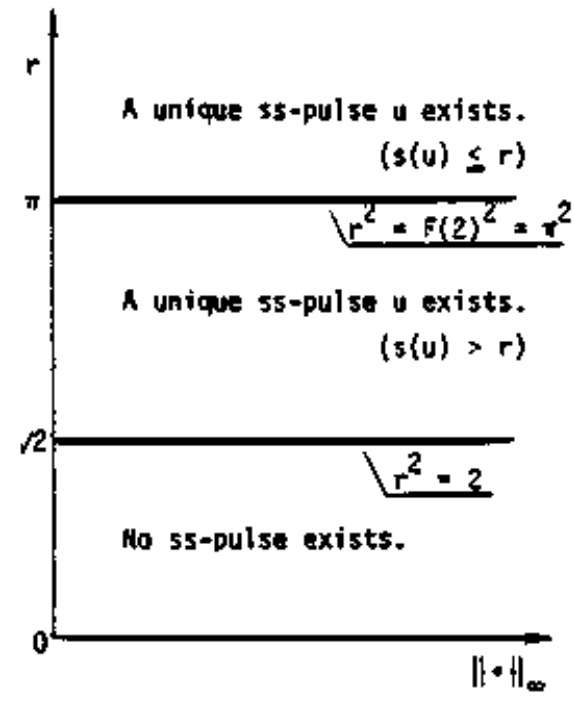

(2) $=2$

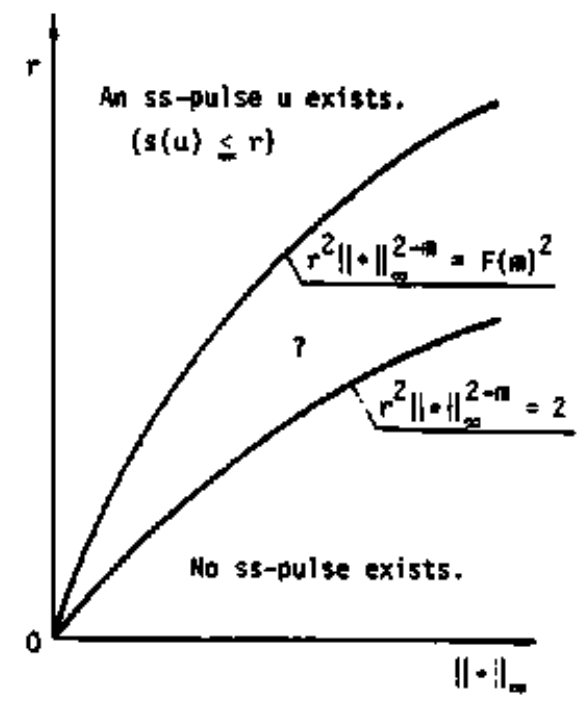

(3) $n>2$

(Figure 8.1) 